\title{
Maximizing Average Sum Rate of Small Cells in 5G Ultra-dense Networks with Deployment Optimization
}

Yihao Luo ( $\sim$ lyh2016@bupt.edu.cn )

Beijing University of Posts and Telecommunications https://orcid.org/0000-0001-5525-3687

\section{Yang Yang}

Beijing University of Posts and Telecommunications

\section{Long Zhang}

Beijing University of Posts and Telecommunications

Dazhong He

Beijing University of Posts and Telecommunications

Jie Yang

Beijing University of Posts and Telecommunications

\section{Research}

Keywords: ultra-dense network, small cell, average sum rate, stochastic geometry, convex optimization

Posted Date: December 15th, 2020

DOI: https://doi.org/10.21203/rs.3.rs-125897/v1

License: (c) (1) This work is licensed under a Creative Commons Attribution 4.0 International License. Read Full License 


\title{
RESEARCH
}

\section{Maximizing Average Sum Rate of Small Cells in 5G Ultra-dense Networks with Deployment Optimization}

\author{
Yihao Luo, Yang Yang*, Long Zhang, Dazhong He and Jie Yang
}

\author{
${ }^{*}$ Correspondence: \\ lyh2016@bupt.edu.cn \\ School of Information and \\ Communication Engineering, \\ Beijing University of Posts and \\ Telecommunications, Beijing, \\ China \\ Full list of author information is \\ available at the end of the article
}

\begin{abstract}
As the evolution of trends of intelligent loT in 5G era, ultra-dense networks (UDN) become a promising paradigm via densely deploying small cells in cellular networks, where the transmission rate of mobile users can be highly improved. In this paper, an investigative study was presented regarding optimizing deployment of small cell base stations (BS) to maximize the average sum rate (ASR) in 5G UDN. In particular on a stochastic geometrical perspective, a homogeneous-type Poisson point process (PPP) was used for depicting an arbitrary arrangement of both macro cell user equipment (UE) and small cell BSs. Moreover, the closed-form probabilities of successful transmission was derived regarding the uplink and downlink of small cells. Then, the ASR of small cells was obtained as well as the problem of maximizing ASR was analyzed with outage constraints. Further, the study also demonstrated that the maximum ASR located in a closed interval of small cell BS density, where the lower and higher bounds of the interval were obtained. Finally, for maximizing the ASR value, the optimal small cell BS density in a closed-form was derived out with convex optimization theory. Simulation analysis indicated that different constraints from the macro cell network led to different maximum ASRs, and interferences caused by small cells and macro cell UE had likewise influenced the performance of small cells.
\end{abstract}

Keywords: ultra-dense network; small cell; average sum rate; stochastic geometry; convex optimization

\section{Introduction}

As the evolution of trends of intelligent IoT (Internet of Things) in $5 \mathrm{G}$ era, the existing network needs to accommodate a larger number of mobile users and communication terminals. Compare to the traditional cellular system, $5 \mathrm{G}$ communication system is going to support $10^{6}$ devices $/ \mathrm{km}^{2}$ connection density and achieve a network throughput up to 1000 times by the year 2020 [1,2]. It is a quite difficult problem for traditional macro base stations (BS) to provide such a huge amount of high-speed traffic and mobile services through limited spectrum resources $[3,4]$. Thus, in the face of these challenges, the concept of ultra-dense networks (UDN) is proposed and becomes a promising paradigm via densely deploying a lot of small cell BSs in cellular networks [5]. UDN allows mobile users to reuse the frequency resources by very closed link distances, which significantly reduces propagation loss and improves transmission gains $[6,7]$. However, ultra-densely deploying small cell BSs will inevitably cause serious interference, which significantly lower the performance of the macro cell networks [8]. 
The premise of high network performance should be based on the effective interference modelling and management. Reference [9] discussed the intracell and intercell interference between macro and small cells, following by the analysis of success probabilities in UDN under both small-scale and large-scale fading. Based on the interference analysis inside small cells, [10] designed an interference mitigation scheme for both intra-tier and cross-tier structures, through utilizing both distributions of orthogonal spectrum and information exchange amongst the small cells. Furthermore, considering the non-orthogonal spectrum sharing, [11] et al. investigated the interference through a stochastic way, to minimize the global and local interference generated by small cells to the macro cell networks. Then, to further exploit local information of interference, a distributed algorithm for users and small cells was proposed in [12], operating the optimal interference management based on maximal interference sets. Nevertheless, the research mentioned above only included the small cells under a fixed topological structure or just analyze the interference characteristic without rate improvement. It is still significant to investigate the performance optimization for small cells under the UDN.

The transmission rate is regarded as an important indicator for the performance of wireless communication systems, which naturally becomes a critical evaluation criterion of small cell deployment in UDN. According to the interference caused by small cells, the transmission rate of the networks can be evaluated for both dedicated and shared spectrum access under fixed grid macro cells [13]. Further, the rate of small cells can be optimized using subchannel and resource management $[14,15]$, caching strategies [16], and joint BS association with power control [17] etc., all of the optimization works above are self-organized and non-cooperative resource allocation. Then, combing with the cooperative resource allocation, the optimization of the transmission rate can be carried out for small cells which are distributed under a single macro BS $[18,19]$. Nevertheless, regarding the evolution of future $5 \mathrm{G}$ system and huge number of wireless communication terminals in UDN, considering only one or several macro cells with a fixed location in the networks is not enough. Therefore, more research works are needed to analyze the transmission rate of UDN with stochastic distributions of users and BSs.

Given network performance optimization of randomly distributed small cells in UDN, we first proposed the model for statistical property of users and BSs by stochastic geometry. Furthermore, small cells' ASR has been maximized by an optimal small cell BS deployment. The research works are based on our previous conference paper accepted by 2018 IEEE International Symposium on Personal from detailed and extended version, Indoor and Mobile Radio Communication (IEEE PIMRC 2018) [20], which includes the extended works as follows:

- Considering the application of stochastic geometry, the modelling of entire networks plays the role of homogeneous Poisson point process (PPP) with small cells sharing uplink frequency resources. On the basis of the transmission powers of small cell BSs and user equipment (UE) can be different, the transmission between small cell user and BS can be divided into uplink and downlink transmissions respectively proposed by this paper.

- Having been derived on a closed-form basis along with the macro cell BS, the Laplace transformation directly contributes to a successful transmission 


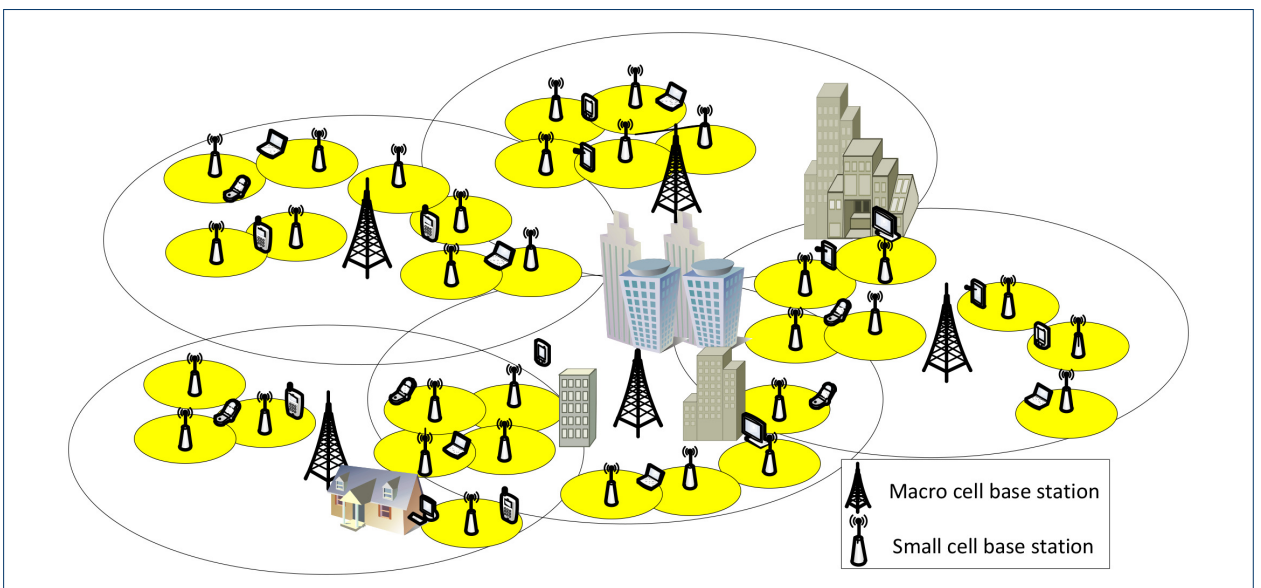

Figure 1 Scenario of UDN

probability. Moreover, such successful transmission with regard to small cells' downlink and uplink communications have been obtained, which contains small cells' interferential influences.

- The ASR is used as the metric for small cells' downlink and uplink communications; it can represent the transmission rate for the unit area in UDN [21]. Then, the problems involving small cells' maximization of ASR as constrained by outage probability can be acquired using a self-variable as small cell BS density $\lambda_{S}$. Moreover, we derive out the interval of $\lambda_{S}$ that maximum ASR value located as the maximization problem can be non-convex. Finally, a closed-form type of optimal BS density amongst small cells is obtained through a series of derivation, resulting in an ASR maximization for these cells.

- Basing from the simulation outcomes considering various considerations in the system, ASR, maximum ASR and outage probability of small cells can be discussed. This verifies that performance of small cells can not only be constrained through macro cell transmission but can also be influenced through small cells' and macro cell UE's interferences.

The rest of our work has been structured by means of the following sections. Section 2 discusses the system model. Section 3 depicts the probabilities for successful transmission for both UDN and small cells' ASR. Section 4 manifests an analysis of the optimization, which comprises the BS density of small cells to determine their maximum ASR. Section 5 provides the simulation results and discussions. Lastly, Section 6 indicates this study's conclusions.

\section{Methods}

\subsection{Scenario and Channel Model}

As Fig.1 shown, a UDN is taken into consideration with several small cells being positioned beneath macro cell systems, from which the uplink may be allocated by the BS of small cells. Considering the UE of macro cells, the model is established to be an autonomous and uniform PPP $\Phi_{M}$ for a 2 D plane $\Re$, with a density of $\lambda_{M}$. Small cell base stations (BS) also correspond to the condition of this PPP on 
$\Re$ that can be defined to be $\Phi_{S}$, with a density of $\lambda_{S}$. Hence, UDN's traffic can be expected to denote a full buffer. Meanwhile, the power for each UE in macro cells, BS in small cells and UE can be expressed to be $P_{M}, P_{S d}$ and $P_{S u}$, respectively.

Macro cell networks can enable the communication in association with the UE and BSs of macro cells to conduct the process involving small cells' downlink and uplink communications, as macro cell networks fulfil the aim of fundamental UDN commercial systems. We set $\operatorname{Pr}_{u}\left(0 \leq \operatorname{Pr}_{u} \leq 1\right)$ as the occurrence probability for small cells' uplink communication. $\operatorname{Pr}_{d}$ is denoted as the occurrence probability for small cells' transmission through uplink; meanwhile, for downlink, it can be denoted as: $\operatorname{Pr}_{d}=1-\operatorname{Pr}_{u}\left(0 \leq \operatorname{Pr}_{d} \leq 1\right)$. On a stochastic geometrical perspective, a typical receiver of small cells can be defined in origin on $\Re[22]$. It will be a small cell UE in case of signal in downlink transmission received by this typical receiver; meanwhile, for an uplink one, it will be a small cell BS as the signal. Then, this typical receiver cannot be affected by statistics of the PPP, following by Sliynyak theory [23]. In the same way, with small cells reusing the macro cell networks' uplink frequency reserves, analysis has been implemented upon a typical receiver - a BS of the macro cell within the UDN. The propagation channel is widely used in the UDN, associating with both path loss and Rayleigh fading, which can be written in the following form:

$$
P_{r}=P_{t} \delta_{t, r} R_{t, r}^{-\alpha}
$$

where $P_{t}$ and $P_{r}$ represent the transmitter power and receiver power, respectively. $R_{t, r}$ denotes the distance between the two power. $\alpha$ indicates the exponent of path loss, satisfying the condition $\alpha \geq 2$. $\delta_{t, r}$ refers to the coefficient of Rayleigh fading, abiding with an autonomous exponential arrangement having a unit mean in each UDN communication association.

\subsection{Average Sum Rate of Small Cells}

The transmission probabilities in UDN have been successfully analyzed considering a system model established in the last section, which has resulted in obtaining ASR of small cells through uplink and downlink transmissions in the network.

\subsubsection{Successful Transmission Probabilities}

What we gave priority to consider in the beginning involves a specification within the conventional BS of macro cells, caused by hindrances for both small cells and macro cell networks within the UDN. Compared with the distance between conventional BS of macro cells and the other hindering small cells, such distance corresponding to the UE and BS of other interfering small cells are negligible due to limited convergence for each small cell [24]. Hence, the signal-to-interference ratio (SIR) for the typical macro cell BS can be alternatively stated to be:

$$
\operatorname{SIR}_{M}=\frac{P_{M} \delta_{M 0} R_{M 0}^{-\alpha}}{\sum_{j \in \Phi_{M}} P_{M} \delta_{j 0} R_{j 0}^{-\alpha}+\sum_{k \in\left(\Phi_{S} \cap A\right)} P_{S u} \delta_{k 0} R_{k 0}^{-\alpha}+\sum_{k \in\left(\Phi_{S} \cap B\right)} P_{S d} \delta_{k 0} R_{k 0}^{-\alpha}},
$$


where $\delta_{M 0}$ and $R_{M 0}$ signify the coefficient of Rayleigh fading and distance from typical BS of the macro cell to the intended UE of macro cells, respectively. In the same way, $\delta_{j 0}$ and $R_{j 0}$ indicate the Rayleigh fading coefficient and distance from the origin of macro cell systems to the node $j$, whist $\delta_{k 0}$ and $R_{k 0}$ are the corresponding parameters of the node $k$ in small cells. Compact point sets $A$ and $B$ respectively satisfy $A=\{m \mid$ node $m$ is the receiver in uplink transmission in small cells $\}$ and $B=\{n \mid$ node $n$ is the receiver in downlink transmission in small cells $\}$.

Denote $I_{M}=\sum_{j \in \Phi_{M}} \delta_{j 0} R_{j 0}^{-\alpha}, I_{S u}=\sum_{k \in\left(\Phi_{S} \cap A\right)} \frac{P_{S u}}{P_{M}} \delta_{k 0} R_{k 0}^{-\alpha}$,

and $I_{S d}=\sum_{k \in\left(\Phi_{S} \cap B\right)} \frac{P_{S d}}{P_{M}} \delta_{k 0} R_{k 0}^{-\alpha}$, eq. (2) can be simplified as:

$$
\operatorname{SIR}_{M}=\frac{\delta_{M 0} R_{M 0}^{-\alpha}}{I_{M}+I_{S u}+I_{S d}}
$$

Following Lemma successfully demonstrates the transmission probability regarding the typical macro cell BS in UDN.

Lemma 1 The successful transmission probability for the typical macro cell BS in UDN satisfies:

$$
\operatorname{Pr}\left(\operatorname{SIR}_{M} \geq \xi_{M}\right)=\exp \left\{-\lambda_{M} \eta_{M}-\lambda_{S} \eta_{M}\left[\operatorname{Pr}_{u}\left(\frac{P_{S u}}{P_{M}}\right)^{\frac{2}{\alpha}}+\operatorname{Pr}_{d}\left(\frac{P_{S d}}{P_{M}}\right)^{\frac{2}{\alpha}}\right]\right\}
$$

where $\operatorname{Pr}(\cdot)$ indicates the probability, $\xi_{M}$ is the SIR threshold of uplink transmission of macro cell UE. $\eta_{M}=\pi R_{M 0}^{2} \xi_{M}^{\frac{2}{\alpha}} \Gamma\left(1+\frac{2}{\alpha}\right) \Gamma\left(1-\frac{2}{\alpha}\right)$, and $\Gamma(\cdot)$ refers to gamma function with the form $\Gamma(z)=\int_{0}^{\infty} e^{-t} t^{z-1} d t$.

From eq. (3), it can be written as:

$$
\operatorname{Pr}\left(\operatorname{SIR}_{M} \geq \xi_{M}\right)=\operatorname{Pr}\left[\delta_{M 0} \geq \xi_{M} R_{M 0}^{\alpha}\left(I_{M}+I_{S u}+I_{S d}\right)\right]
$$

With $\xi_{M}$ fulfiling the constraint of the autonomous exponential arrangement using the unit mean, eq. (5) may be alternatively stated to be:

$$
\begin{aligned}
& \operatorname{Pr}\left(\operatorname{SIR}_{M} \geq \xi_{M}\right)=E\left[e^{-\xi_{M} R_{M 0}^{\alpha}\left(I_{M}+I_{S u}+I_{S d}\right)}\right] \\
& =\mathcal{L}_{I_{M}}\left(\xi_{M} R_{M 0}^{\alpha}\right) \mathcal{L}_{I_{S u}}\left(\xi_{M} R_{M 0}^{\alpha}\right) \mathcal{L}_{I_{S d}}\left(\xi_{M} R_{M 0}^{\alpha}\right)
\end{aligned}
$$

where $\mathcal{L}_{I_{M}}(\cdot), \mathcal{L}_{I_{S u}}(\cdot)$ and $\mathcal{L}_{I_{S d}}(\cdot)$ are Laplace transformation of $I_{M}, I_{S u}$ and $I_{S d}$, respectively. All of them can be satisfied as:

$$
\begin{aligned}
& \mathcal{L}_{I_{M}}\left(\xi_{M} R_{M 0}^{\alpha}\right)=\exp \left[-\lambda_{M} \int_{0}^{\infty} E_{\delta_{j 0}}\left(1-e^{-\xi_{M} R_{M 0}^{\alpha} r^{-\alpha}}\right) d r\right] \\
& =\exp \left[-\lambda_{M} \pi R_{M 0}^{2} \xi_{M}^{\frac{2}{\alpha}} \Gamma\left(1+\frac{2}{\alpha}\right) \Gamma\left(1-\frac{2}{\alpha}\right)\right], \\
& \mathcal{L}_{I_{S u}}\left(\xi_{M} R_{M 0}^{\alpha}\right)=\exp \left[-\lambda_{S} \operatorname{Pr}_{u}\left(\frac{P_{S u}}{P_{M}}\right)^{\frac{2}{\alpha}} \pi R_{M 0}^{2} \xi_{M}^{\frac{2}{\alpha}} \Gamma\left(1+\frac{2}{\alpha}\right) \Gamma\left(1-\frac{2}{\alpha}\right)\right],
\end{aligned}
$$




$$
\mathcal{L}_{I_{S d}}\left(\xi_{M} R_{M 0}^{\alpha}\right)=\exp \left[-\lambda_{S} \operatorname{Pr}_{d}\left(\frac{P_{S d}}{P_{M}}\right)^{\frac{2}{\alpha}} \pi R_{M 0}^{2} \xi_{M}^{\frac{2}{\alpha}} \Gamma\left(1+\frac{2}{\alpha}\right) \Gamma\left(1-\frac{2}{\alpha}\right)\right]
$$

Denote $\eta_{M}=\pi R_{M 0}^{2} \xi_{M}^{\frac{2}{\alpha}} \Gamma\left(1+\frac{2}{\alpha}\right) \Gamma\left(1-\frac{2}{\alpha}\right)$, so eq. (4) is obtained. Similarly, the SIR at the typical small cell UE in uplink transmission satisfies:

$$
\operatorname{SIR}_{S u}=\frac{P_{S u} \delta_{S u 0} R_{S u 0}^{-\alpha}}{\sum_{j \in \Phi_{M}} P_{M} \delta_{j 0} R_{j 0}^{-\alpha}+\sum_{k \in\left(\Phi_{S} \cap A\right)} P_{S u} \delta_{k 0} R_{k 0}^{-\alpha}+\sum_{k \in\left(\Phi_{S} \cap B\right)} P_{S d} \delta_{k 0} R_{k 0}^{-\alpha}}
$$

where $\delta_{S u 0}$ and $R_{S u 0}$ mean the coefficient of Rayleigh fading and distance from the typical UE of the small cell to its serving BS, respectively. Hence, the SIR of a typical BS in small cell for a downlink communication is denoted as:

$$
\operatorname{SIR}_{S d}=\frac{P_{S d} \delta_{S d 0} R_{S d 0}^{-\alpha}}{\sum_{j \in \Phi_{M}} P_{M} \delta_{j 0} R_{j 0}^{-\alpha}+\sum_{k \in\left(\Phi_{S} \cap A\right)} P_{S u} \delta_{k 0} R_{k 0}^{-\alpha}+\sum_{k \in\left(\Phi_{S} \cap B\right)} P_{S d} \delta_{k 0} R_{k 0}^{-\alpha}}
$$

where $\delta_{S d 0}$ and $R_{S d 0}$ correspondingly signify the coefficient of Rayleigh fading and distance from typical BS of small cell to intended UE. Here $\xi_{S u}$ and $\xi_{S d}$ are indicated to be the respective SIR tolerances for small cells' uplink and downlink communication. Then two Lemmas can be obtained as follows:

Lemma 2 The successful transmission probability for the typical small cell UE in uplink transmission satisfies:

$$
\operatorname{Pr}\left(\operatorname{SIR}_{S u} \geq \xi_{S u}\right)=\exp \left\{-\lambda_{M} \eta_{S u}\left(\frac{P_{M}}{P_{S u}}\right)^{\frac{2}{\alpha}}-\lambda_{S} \eta_{S u}\left[\operatorname{Pr}_{u}+\operatorname{Pr}_{d}\left(\frac{P_{S d}}{P_{S u}}\right)^{\frac{2}{\alpha}}\right]\right\}
$$

Where $\eta_{S u}=\pi R_{S u 0}^{2} \xi_{S u}^{\frac{2}{\alpha}} \Gamma\left(1+\frac{2}{\alpha}\right) \Gamma\left(1-\frac{2}{\alpha}\right)$.

Following the similar steps as Lemma 1, eq. (12) can be obtained.

Lemma 3 The successful transmission probability for the typical small cell BS in downlink transmission satisfies:

$$
\operatorname{Pr}\left(\operatorname{SIR}_{S d} \geq \xi_{S d}\right)=\exp \left\{-\lambda_{M} \eta_{S d}\left(\frac{P_{M}}{P_{S d}}\right)^{\frac{2}{\alpha}}-\lambda_{S} \eta_{S d}\left[\operatorname{Pr}_{u}\left(\frac{P_{S u}}{P_{S d}}\right)^{\frac{2}{\alpha}}+\operatorname{Pr}_{d}\right]\right\}
$$

where $\eta_{S d}=\pi R_{S d 0}^{2} \xi_{S d}^{\frac{2}{\alpha}} \Gamma\left(1+\frac{2}{\alpha}\right) \Gamma\left(1-\frac{2}{\alpha}\right)$.

Following the similar steps as Lemma 1, eq. (13) can be obtained. 
2.2.2 Average Sum Rate of uplink and downlink Communications in Small Cells Based on the work in [25], the ASR of small cells can be defined as:

$$
f_{S n}\left(\lambda_{S}\right)=\operatorname{Pr}_{n} \lambda_{S} \overline{R_{S n}} \quad(n \in\{u, d\}),
$$

where $\overline{R_{S n}}$ with subscript $n \in\{u, d\}$ correspondingly represents mean uplink rates ( $n$ is ' $u$ ') and transmission through downlink ( $n$ is ' $d$ '). $\overline{R_{S n}}$ satisfies its detailed configuration as follow [26]:

$$
\overline{R_{S n}}=\sup _{\xi_{S n} \geq 0} W \log _{2}\left(1+\xi_{S n}\right) \operatorname{Pr}\left(\operatorname{SIR}_{S n} \geq \xi_{S n}\right) \quad(n \in\{u, d\}),
$$

where $W$ represents bandwidth for the uplink transmission of macro cells, being reused by small cells. Since $\xi_{S u}$ or $\xi_{S d}$ in eq. (15) has been already defined before, the following definition can be obtained based on Lemma 2 and $\mathbf{3}$.

Definition 1 The ASR of uplink transmission in small cells is

$$
\begin{aligned}
& f_{S u}\left(\lambda_{S}\right)=W \operatorname{Pr}_{u} \lambda_{S} \log _{2}\left(1+\xi_{S u}\right) \times \\
& \exp \left\{-\lambda_{M} \eta_{S u}\left(\frac{P_{M}}{P_{S u}}\right)^{\frac{2}{\alpha}}-\lambda_{S} \eta_{S u}\left[\operatorname{Pr}_{u}+\operatorname{Pr}_{d}\left(\frac{P_{S d}}{P_{S u}}\right)^{\frac{2}{\alpha}}\right]\right\},
\end{aligned}
$$

while the ASR of downlink transmission in small cells can be expressed as

$$
\begin{aligned}
& f_{S d}\left(\lambda_{S}\right)=W \operatorname{Pr}_{d} \lambda_{S} \log _{2}\left(1+\xi_{S d}\right) \times \\
& \exp \left\{-\lambda_{M} \eta_{S d}\left(\frac{P_{M}}{P_{S d}}\right)^{\frac{2}{\alpha}}-\lambda_{S} \eta_{S d}\left[\operatorname{Pr}_{u}\left(\frac{P_{S u}}{P_{S d}}\right)^{\frac{2}{\alpha}}+\operatorname{Pr}_{d}\right]\right\}
\end{aligned}
$$

Denoting function of small cell density $f\left(\lambda_{S}\right)$ as the ASR of small cells in UDN, hence it satisfies:

$$
f\left(\lambda_{S}\right)=f_{S u}\left(\lambda_{S}\right)+f_{S d}\left(\lambda_{S}\right)
$$

\subsection{Optimization of ASR for Small Cells}

As small cells have reused the frequency resources from the systems of macro cells, the reliable transmission of macro cells cannot be disturbed. Specifically, small cell transmission should warrant the outage probability of BS of macro cells in UDN. Considering the division of small cell transmission as either downlink or uplink, the limitations are provided as follows:

$$
\begin{aligned}
& 0 \leq \lambda_{S} \leq \lambda_{S, \max }, \\
& 1-e^{-\lambda_{M} \eta_{M}-\lambda_{S} \eta_{M}\left[\operatorname{Pr}_{u}\left(\frac{P_{S u}}{P_{M}}\right)^{\frac{2}{\alpha}}+\operatorname{Pr}_{d}\left(\frac{P_{S d}}{P_{M}}\right)^{\frac{2}{\alpha}}\right]} \leq \theta_{M},
\end{aligned}
$$




$$
\begin{aligned}
& 1-e^{-\lambda_{M} \eta_{S u}\left(\frac{P_{M}}{P_{S u}}\right)^{\frac{2}{\alpha}}-\lambda_{S} \eta_{S u}\left[\operatorname{Pr}_{u}+\operatorname{Pr}_{d}\left(\frac{P_{S d}}{P_{S u}}\right)^{\frac{2}{\alpha}}\right]} \leq \theta_{S u}, \\
& 1-e^{-\lambda_{M} \eta_{S d}\left(\frac{P_{M}}{P_{S d}}\right)^{\frac{2}{\alpha}}-\lambda_{S} \eta_{S d}\left[\operatorname{Pr}_{u}\left(\frac{P_{S u}}{P_{S d}}\right)^{\frac{2}{\alpha}}+\operatorname{Pr}_{d}\right]} \leq \theta_{S d},
\end{aligned}
$$

where $\lambda_{S, \max }$ represents the highest density of the BS of small cells in UDN. $\theta_{M}$, $\theta_{S u}$ and $\theta_{S d}\left(\theta_{M}, \theta_{S u}, \theta_{S d} \in(0,1]\right)$ respectively denote outage probability thresholds for macro cell BS, small cell BS and UE. Therefore, the ASR optimization problem of small cells can be obtained:

$$
\begin{aligned}
& \max \quad f\left(\lambda_{S}\right), \\
& \text { s.t. } \quad(19), \quad(20), \quad(21), \quad(22) \text {. }
\end{aligned}
$$

Based on inequality (20), we have:

$$
\lambda_{S} \leq \frac{-\lambda_{M} P_{M}^{\frac{2}{\alpha}}-\frac{P_{M}^{\frac{2}{\alpha}}}{\eta_{M}} \ln \left(1-\theta_{M}\right)}{\operatorname{Pr}_{u} P_{S u}^{\frac{2}{\alpha}}+\operatorname{Pr}_{d} P_{S d}^{\frac{2}{\alpha}}}
$$

From inequality (21), we have:

$$
\lambda_{S} \leq \frac{-\lambda_{M} P_{M}^{\frac{2}{\alpha}}-\frac{P_{S u}^{\frac{2}{\alpha}}}{\eta_{S u}} \ln \left(1-\theta_{S u}\right)}{\operatorname{Pr}_{u} P_{S u}^{\frac{2}{\alpha}}+\operatorname{Pr}_{d} P_{S d}^{\frac{2}{\alpha}}}
$$

From inequality (22), we have:

$$
\lambda_{S} \leq \frac{-\lambda_{M} P_{M}^{\frac{2}{\alpha}}-\frac{P_{S d}^{\frac{2}{\alpha}}}{\eta_{S d}} \ln \left(1-\theta_{S d}\right)}{\operatorname{Pr}_{u} P_{S u}^{\frac{2}{\alpha}}+\operatorname{Pr}_{d} P_{S d}^{\frac{2}{\alpha}}}
$$

Denote $\lambda_{S, \sup 1}=\frac{-\lambda_{M} P_{M}^{\frac{2}{\alpha}}-\frac{P_{M}^{\frac{2}{\alpha}}}{\eta_{M}} \ln \left(1-\theta_{M}\right)}{\operatorname{Pr}_{u} P_{S u}^{\frac{2}{\alpha}}+\operatorname{Pr}_{d} P_{S d}^{\frac{2}{\alpha}}}, \lambda_{S, \sup 2}=\frac{-\lambda_{M} P_{M}^{\frac{2}{\alpha}}-\frac{P_{S u}^{\frac{2}{\alpha}}}{\eta_{S u}} \ln \left(1-\theta_{S u}\right)}{\operatorname{Pr}_{u} P_{S u}^{\frac{2}{\alpha}}+\operatorname{Pr}_{d} P_{S d}^{\frac{2}{\alpha}}}$, $\lambda_{S, \sup 3}=\frac{-\lambda_{M} P_{M}^{\frac{2}{\alpha}}-\frac{P_{S d}^{\frac{2}{\alpha}}}{\eta_{S d}} \ln \left(1-\theta_{S d}\right)}{\operatorname{Pr}_{u} P_{S u}^{\frac{2}{\alpha}}+\operatorname{Pr}_{d} P_{S d}^{\frac{2}{\alpha}}}$. So according to (19), the upper density bound of small cells satisfies $\lambda_{S \text {,sup }}=\left\{\lambda_{S, \max }, \lambda_{S \text {, sup } 1}, \lambda_{S, \text { sup } 2}, \lambda_{S, \text { sup } 3}\right\}$.

Let $A_{1}=W \operatorname{Pr}_{u} \log _{2}\left(1+\xi_{S u}\right), A_{2}=\lambda_{M} \eta_{S u}\left(\frac{P_{M}}{P_{S u}}\right)^{\frac{2}{\alpha}}, A_{3}=\eta_{S u}\left[\operatorname{Pr}_{u}+\operatorname{Pr}_{d}\left(\frac{P_{S d}}{P_{S u}}\right)^{\frac{2}{\alpha}}\right]$, $B_{1}=W \operatorname{Pr}_{d} \log _{2}\left(1+\xi_{S d}\right), B_{2}=\lambda_{M} \eta_{S d}\left(\frac{P_{M}}{P_{S d}}\right)^{\frac{2}{\alpha}}, B_{3}=\eta_{S d}\left[\operatorname{Pr}_{u}\left(\frac{P_{S u}}{P_{S d}}\right)^{\frac{2}{\alpha}}+\operatorname{Pr}_{d}\right]$.

The ASR of small cells can be written as: 


$$
f\left(\lambda_{S}\right)=A_{1} \lambda_{S} e^{-A_{2}-\lambda_{S} A_{3}}+B_{1} \lambda_{S} e^{-B_{2}-\lambda_{S} B_{3}},
$$

From (27), we can see that $f\left(\lambda_{S}\right)$ is not a convex function [27]. However, as $f\left(\lambda_{S}\right)$ is composed by ASRs for small cells' transmission through both downlink and uplink, we have a proposition about the maximum value of function $f\left(\lambda_{S}\right)$, as specified below.

Proposition 1 The maximum value of function $f\left(\lambda_{S}\right)$ locates at the interval $\left[\lambda_{S, l}, \lambda_{S, h}\right]$, where $\lambda_{S, l}=\min \left\{\frac{1}{A_{3}}, \frac{1}{B_{3}}\right\}$ and $\lambda_{S, h}=\max \left\{\frac{1}{A_{3}}, \frac{1}{B_{3}}\right\}$.

According to (16), (17), we know $f_{S u}\left(\lambda_{S}\right)=A_{1} \lambda_{S} e^{-A_{2}-\lambda_{S} A_{3}}, f_{S d}\left(\lambda_{S}\right)=$ $B_{1} \lambda_{S} e^{-B_{2}-\lambda_{S} B_{3}}$. Take the first derivative of both $f_{S u}\left(\lambda_{S}\right)$ and $f_{S d}\left(\lambda_{S}\right)$, we have:

$$
\begin{aligned}
& \frac{d\left[f_{S u}\left(\lambda_{S}\right)\right]}{d \lambda_{S}}=A_{1} e^{-A_{2}-\lambda_{S} A_{3}}\left(1-\lambda_{S} A_{3}\right), \\
& \frac{d\left[f_{S d}\left(\lambda_{S}\right)\right]}{d \lambda_{S}}=B_{1} e^{-B_{2}-\lambda_{S} B_{3}}\left(1-\lambda_{S} B_{3}\right),
\end{aligned}
$$

make $\frac{d\left[f_{S u}\left(\lambda_{S}\right)\right]}{d \lambda_{S}}=0$ and $\frac{d\left[f_{S d}\left(\lambda_{S}\right)\right]}{d \lambda_{S}}=0$, the maximum values of $f_{S u}\left(\lambda_{S}\right)$ and $f_{S d}\left(\lambda_{S}\right)$ are $\frac{1}{A_{3}}$ and $\frac{1}{B_{3}}$, respectively. Also we know when $\lambda_{S} \in\left[0, \frac{1}{A_{3}}\right)$, function $f_{S u}\left(\lambda_{S}\right)$ increases monotonically, and $f_{S u}\left(\lambda_{S}\right)$ decreases monotonically when $\lambda_{S} \in\left(\frac{1}{A_{3}},+\infty\right)$. Similarly, function $f_{S d}\left(\lambda_{S}\right)$ increases monotonically when $\lambda_{S} \in\left[0, \frac{1}{B_{3}}\right)$, and it decreases monotonically with $\lambda_{S} \in\left(\frac{1}{B_{3}},+\infty\right)$.

Denote $\lambda_{S, l}=\min \left\{\frac{1}{A_{3}}, \frac{1}{B_{3}}\right\}, \lambda_{S, h}=\max \left\{\frac{1}{A_{3}}, \frac{1}{B_{3}}\right\}$. Without loss of generality, suppose $\lambda_{S, l}=\frac{1}{A_{3}}$, and $\lambda_{S, h}=\frac{1}{B_{3}}$. According to the results before, for $\forall \lambda_{S} \in$ $\left[0, \frac{1}{A_{3}}\right)$, it can be known that $f_{S u}\left(\lambda_{S}\right)<f_{S u}\left(\frac{1}{A_{3}}\right)$, and $f_{S d}\left(\lambda_{S}\right)<f_{S d}\left(\frac{1}{A_{3}}\right)$ because $\lambda_{S}<\frac{1}{A_{3}}<\frac{1}{B_{3}}$. So we can get:

$$
f\left(\lambda_{S}\right)<f\left(\frac{1}{A_{3}}\right) \quad \text { when } \quad \lambda_{S} \in\left[0, \frac{1}{A_{3}}\right)
$$

Similarly, for $\forall \lambda_{S} \in\left(\frac{1}{B_{3}},+\infty\right)$, it can be known that $f_{S u}\left(\lambda_{S}\right)<f_{S u}\left(\frac{1}{B_{3}}\right)$ for $\frac{1}{A_{3}}<\frac{1}{B_{3}}<\lambda_{S}$, and $f_{S d}\left(\lambda_{S}\right)<f_{S d}\left(\frac{1}{B_{3}}\right)$. Also we can get:

$$
f\left(\lambda_{S}\right)<f\left(\frac{1}{A_{3}}\right) \text { when } \lambda_{S} \in\left(\frac{1}{B_{3}},+\infty\right)
$$

The same results can be worked out if we define $\lambda_{S, l}=\frac{1}{B_{3}}$, and $\lambda_{S, h}=\frac{1}{A_{3}}$. Thus, the maximum value of function $f\left(\lambda_{S}\right)$ must located at the interval $\left[\lambda_{S, l}, \lambda_{S, h}\right]$. Based on Proposition 1, following theorem shows the small cells' optimal density, enabling their maximum ASR under power and outage probability limitations. 
Theorem 1 The optimal small cell density $\lambda_{S}^{\max }$ for maximizing ASR of small cells satisfies:

$$
\lambda_{S}^{\max }=\left\{\begin{array}{cc}
\lambda_{S, \text { sup }} & \lambda_{S, \text { sup }}<\lambda_{S}^{*} \\
\lambda_{S}^{*} & \lambda_{S}^{*} \leq \lambda_{S, \text { sup }}
\end{array},\right.
$$

where $\lambda_{S}^{*}$ satisfies

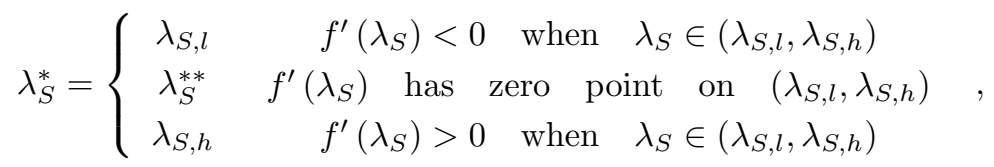

where $f^{\prime}\left(\lambda_{S}\right)$ refers to the first derivative of $f\left(\lambda_{S}\right)$ in terms of $\lambda_{S}$, $\lambda_{S}^{* *}=\underset{\lambda_{S} \in\left\{\frac{1}{A_{3}}, \lambda_{S}^{* * *}, \frac{1}{B_{3}}\right\}}{\arg \max } f\left(\lambda_{S}\right)$ and $\lambda_{S}^{* * *}$ is the solution of $f^{\prime}\left(\lambda_{S}\right)=0$ when $f^{\prime}\left(\lambda_{S}\right)$ has zero point on $\left(\lambda_{S, l}, \lambda_{S, h}\right)$. From (28) and (29), we know the first derivative of $f\left(\lambda_{S}\right)$ in terms of $\lambda_{S}$ is:

$$
f^{\prime}\left(\lambda_{S}\right)=A_{1} e^{-A_{2}-\lambda_{S} A_{3}}\left(1-\lambda_{S} A_{3}\right)+B_{1} e^{-B_{2}-\lambda_{S} B_{3}}\left(1-\lambda_{S} B_{3}\right),
$$

Firstly, if $f^{\prime}\left(\lambda_{S}\right)<0$, with reference to Proposition 1, it is deduced that $f\left(\lambda_{S}\right)$ may result to a maximum value as $\lambda_{S}=\lambda_{S, l}$ because $f\left(\lambda_{S}\right)$ is monotonically decreased on $\left(\lambda_{S, l}, \lambda_{S, h}\right)$. Secondly, if $f^{\prime}\left(\lambda_{S}\right)>0, f\left(\lambda_{S, h}\right)$ is the maximum value since $f\left(\lambda_{S}\right)$ has been uniformly enhanced on $\left(\lambda_{S, l}, \lambda_{S, h}\right)$. Thirdly, considering $f\left(\lambda_{S}\right)$ to be a constantly bounded function on close-set $\left[\lambda_{S, l}, \lambda_{S, h}\right]$, and denoting $\exists \lambda_{S}^{* * *} \in$ $\left(\lambda_{S, l}, \lambda_{S, h}\right)$ as leading to $f^{\prime}\left(\lambda_{S}^{* * *}\right)=0, f\left(\lambda_{S}^{* * *}\right)$ represents the local maximum or minimum value on $\left[\lambda_{S, l}, \lambda_{S, h}\right]$. Thus, $f\left(\lambda_{S}^{* *}\right)$ represents the maximum value when $\lambda_{S}^{* *}=\underset{\lambda_{S} \in\left\{\frac{1}{A_{3}}, \lambda_{S}^{* * *}, \frac{1}{B_{3}}\right\}}{\arg \max } f\left(\lambda_{S}\right)$. Finally, combining those three points above, $\lambda_{S}^{*}$ can be obtained. The optimal small cell density $\lambda_{S}^{\max }$ with consideration of small cells' ASR maximization can be depicted by the equation, with involving both power and outage probability limitations for transmission by both small and large cells (32).

Remark 1 A special situation is when $P_{S u}=P_{S d}, \operatorname{Pr}_{u}=\operatorname{Pr}_{d}=0.5, \xi_{S u}=\xi_{S d}$, $R_{S u 0}=R_{S d 0}$, then $A_{3}=B_{3}=\eta_{S u}=\eta_{S d}$. So the feasible region of small cell $B S$ density becomes one point, and the ASR of small cells gets its peak value at $\lambda_{S}^{*}=\lambda_{S, l}=\lambda_{S, h}$. Let $C_{3}=\frac{1}{A_{3}}$. Eventually the optimal small cell density $\lambda_{S}^{\max }$ can be written as

$$
\lambda_{S}^{\max }=\left\{\begin{array}{cc}
\lambda_{S, \text { sup }} & \lambda_{S, \text { sup }}<\lambda_{S}^{*} \\
\lambda_{S}^{*} & \lambda_{S}^{*} \leq \lambda_{S, \text { sup }}
\end{array},\right.
$$

where $\lambda_{S}^{*}=C_{3}$. 
Table 1 Network Simulation Parameters

\begin{tabular}{ccc}
\hline Symbol & Physical meanings & Value \\
\hline$\alpha$ & Path loss & 4 \\
$\lambda_{M}$ & Density of macro cell UE & $1 \times 10^{-6}$ macro cell UE $/ \mathrm{m}^{2}$ \\
$\lambda_{S}$ & Density of small cell BSs & $1 \times 10^{-5}$ small cell BS $/ \mathrm{m}^{2}$ \\
$P_{M}$ & Transmit power of macro cell UE & $35 \mathrm{dBm}$ \\
$P_{S u}$ & Transmit power of small cell UE & $25 \mathrm{dBm}$ \\
$P_{S d}$ & Transmit power of small cell BS & $30 \mathrm{dBm}$ \\
$\operatorname{Pr}_{u} / \operatorname{Pr}_{d}$ & uplink/downlink transmission probability & $0.6 / 0.4$ \\
$\xi_{M}$ & SIR threshold of the macro cell uplink transmission & $0 \mathrm{~dB}$ \\
$\xi_{S u}$ & SIR threshold of the small cell uplink transmission & $0 \mathrm{~dB}$ \\
$\xi_{S d}$ & SIR threshold of the small cell downlink transmission & $0 \mathrm{~dB}$ \\
$R_{M 0}$ & Distance between macro cell BS and the typical cellular user & $100 \mathrm{~m}$ \\
$R_{S u 0}$ & Distance between small cell UE and the typical small cell BS & $50 \mathrm{~m}$ \\
$R_{S d 0}$ & Distance between small cell BS and the typical small cell UE & $60 \mathrm{~m}$ \\
\hline
\end{tabular}

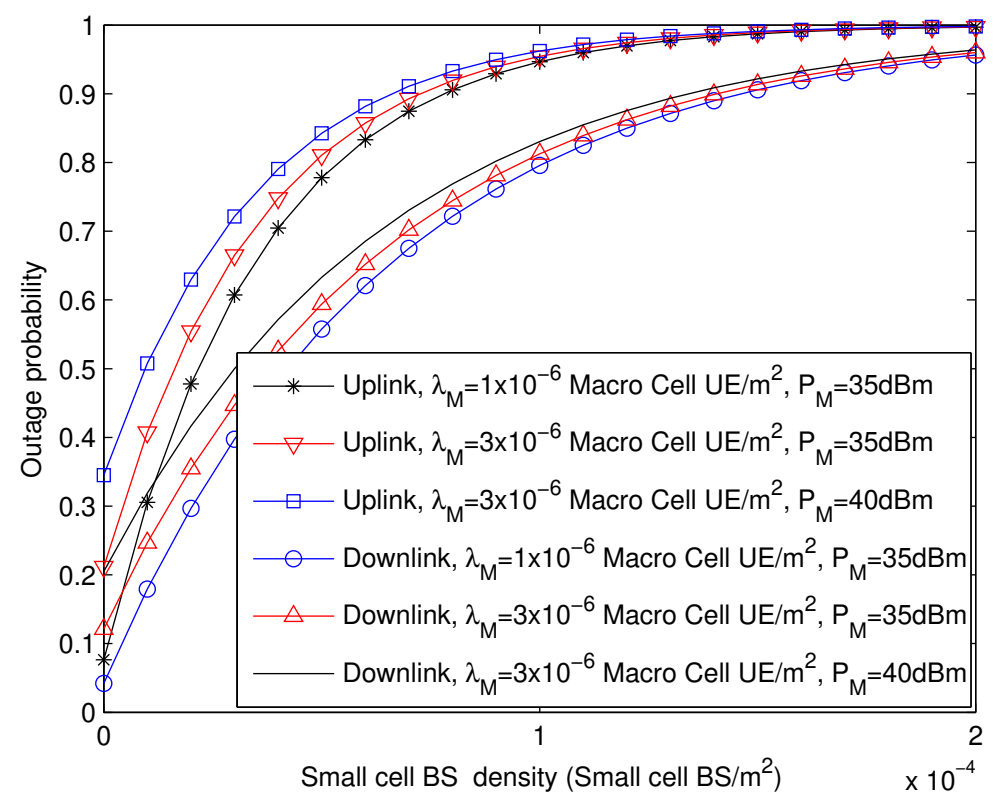

Figure 2 Outage probability in small cells

\section{Results and Discussion}

By means of simulation, this section presents the evaluation of small cells' ASR. Table I indicates the primary simulation conditions.

In Fig. 2, we exhibit the outage probability of small cells varying against the increase of small cell BS density. Firstly, we can see the outage probabilities containing both uplink and downlink transmission grow up when small cell BS density increases. This is caused by the fact that high small cell BS density produces more serious interference to the entire networks. Secondly, it is clear that the outage probabilities of small cells are increased with higher density of macro cell UE. The finding to explain this phenomenon is that high interference to the small cells are generated by high density of macro cell UE, which also determines tough condition relating to constraints to the communications inside small cells. In addition, the outage probability ascends as the transmission power of macro cell UE goes up to $40 \mathrm{dBm}$, enhancing the high intensity of interference to the small cells. Thirdly, the 
uplink transmit power is smaller than the downlink transmit power in small cells, which means the small cell UE get more serious interference as the receivers in downlink communication, so we can see outage probability of downlink communication is lower than that of uplink communication. Last, the uplink and downlink probabilities in small cells are configured to 0.6 and 0.4 , respectively. We can know uplink transmission in small cells will cause more interference in the UDN as the small cell BS density increases. Therefore, the uplink outage probability rises relatively faster than the downlink outage probability.

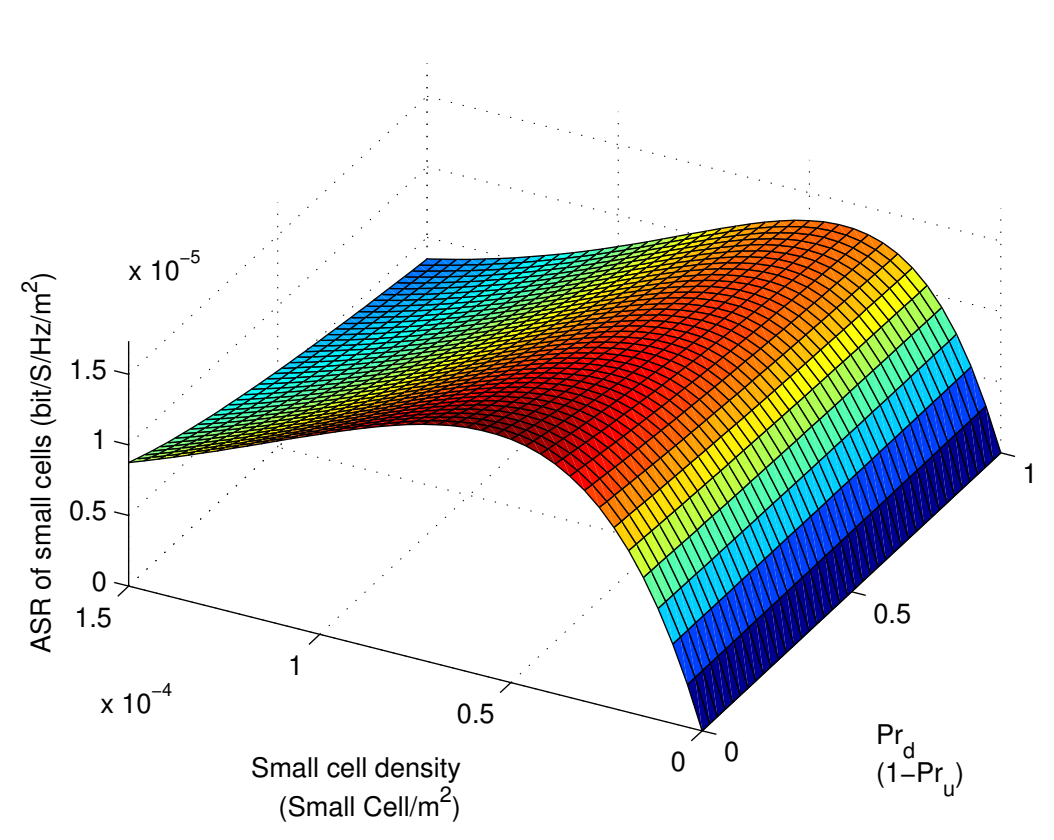

Figure 3 ASR of small cells vs. small cell density and probability of downlink

In Fig. 3, ASR of small cells is shown with various small cell BS density and probability of downlink transmission. It is noted that the influence of probability of uplink transmission can also be seen from this figure because $\operatorname{Pr}_{d}=1-\operatorname{Pr}_{u}$. Here we set $25 \mathrm{dBm}$ as the power of both small cell BS and UE. Then, under the same density of small cells, we can see when $\operatorname{Pr}_{d}=0$, the value of ASR is higher than the one when $\operatorname{Pr}_{d}=1$. The reason to demonstrate this is that $R_{S d 0}$ is larger than $R_{S u 0}$ in our simulation, i.e., the signal of downlink transmission will be in the process more propagation loss because the downlink communication distance is much longer compared to uplink communication. Fig. 3 also illustrates that in the case of low small cell BS density, the ASR rises when the density of small cell BS increases, which means the performance of UDN can be improved through small cell BSs. Nevertheless, we can see as the small cell BS density grows up continually, the interference produced by small cells in the UDN should not be neglected, which causes the ASR decreases.

Fig. 4 and Fig. 5 show the ASR of small cells with two different feasible regions of small cell BS density. The maximum uplink ASR and maximum downlink ASR of small cells are also shown in both two figures. The maximum ASR of small cells, 


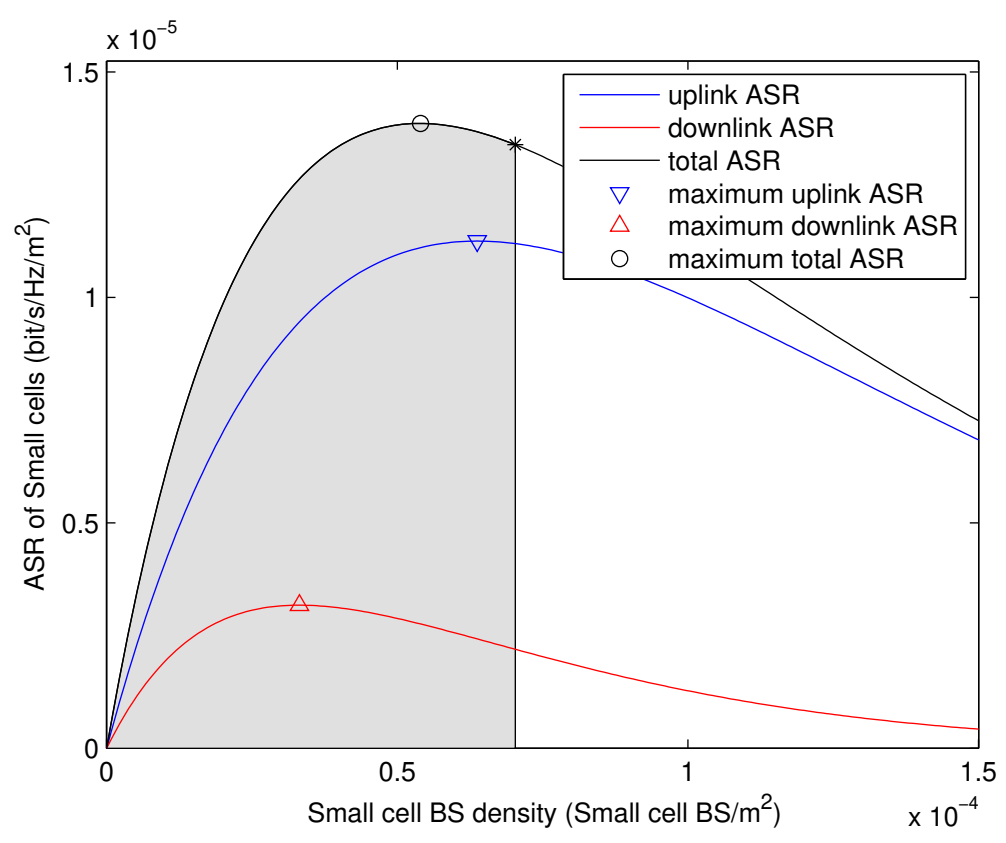

Figure 4 ASR of small cells vs. small cell BS density (Maximum ASR can be obtained)

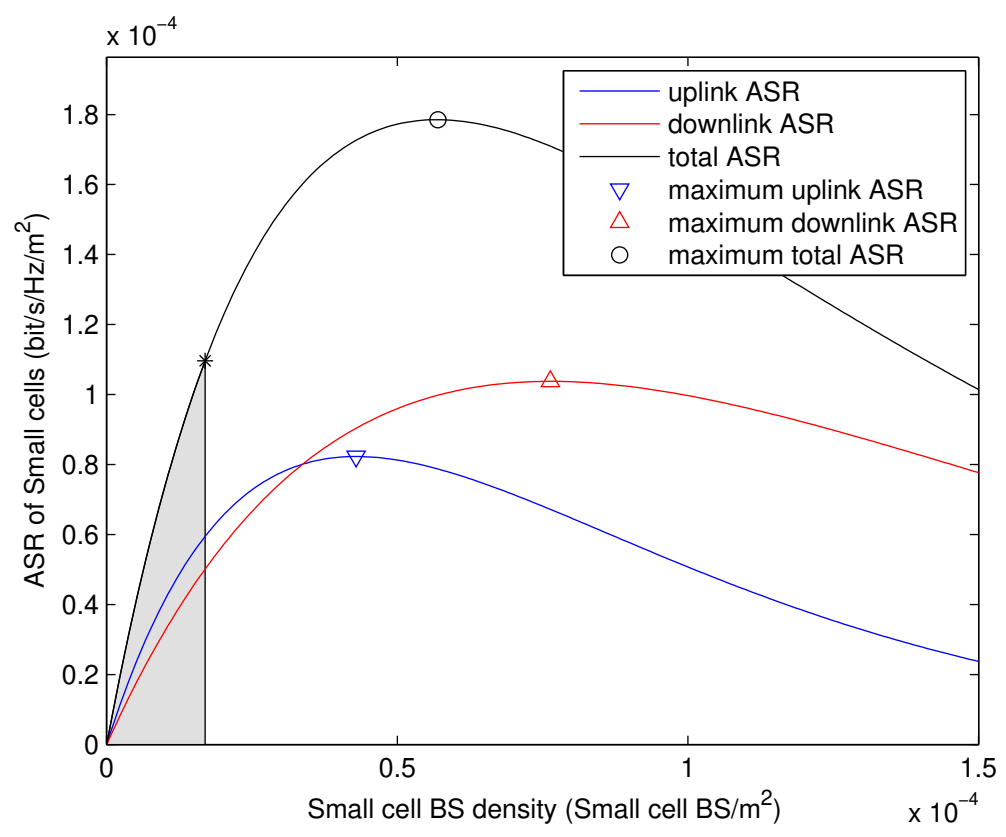

Figure 5 ASR of small cells vs. small cell BS density (Boundary value of ASR can be obtained) 
which is the summation of the two ASRs mentioned before, locate between those two ASRs at the interval with respect to small cell BS density. This phenomenon exactly verifies Proposition 1. Being similar to Fig. 3, here the ASR value initially rises then falls as the small cell BS density increases because the interference generated by small cells is not negligible when the density is high. In Fig. 4, the power of small cell UE and BS are set as $14 \mathrm{dbm}$ and $11 \mathrm{dbm}$, respectively. It is apparent that downlink ASR of small cells is lower than uplink ASR at given scope of small cell BS density, which means that the receivers in downlink transmission of small cells can be greatly affected by interference compared to uplink receivers. What is more, because the power of small cell UE and BS is low, macro cells can allow more small cells to reuse their frequency resources. As a result, it is obvious that the feasible region of small cell BS density is wide, and the value for maximum ASR of small cells can be obtained.

Similarly, in Fig. 5, the power of small cell UE and BS are set as 25dbm and 30dbm, respectively. So we can see that the downlink ASR is higher than the uplink ASR with respect to number for ASR of small cells at the given range of small cell BS density, but the growth of downlink ASR is slighter compared to growth of uplink when small cell BS density is low. The reason why makes this happen is that the uplink transmission is shorter than downlink transmission in the distance, which results in less propagation loss for the uplink signal compared to the downlink signals. Besides, the power for both small cell UE and BS are high so that the feasible region of small cell BS density is compressed, because small cells cannot cause enormous interference to the transmission of macro cells. Thus, only boundary value of the feasible region can be obtained.

Fig. 6 reveals the existing association of the density of the UE of macro cells and maximum ASR, in which the latter decreases as the former increases. It is noted that high intensity of interference caused by high macro cell UE density will exert on the small cells, thereby leading to a high standard for constraints to small cells. In the case of exceedingly high UE density, the receivers in small cells severely suffer from macro cell UE due to interference, which will lead ASR values to zero. However, in the case of extremely low UE density, macro cell BS can resist the high intensity of interference at the effect of higher power of macro cell UE. Then the optimal value of small cell BS density can be obtained in the UDN. Additionally, the boundary value of feasible region can be obtained based on the optimum densities of the BS of small cells under harsh condition concerning the small cells' limitations, with continually increasing the macro cell UE density. Roughly, there is a linear decrease in small cells' maximum ASR.

Fig. 7 demonstrates the change in small cells' maximum ASR following a rise in macro cell power. The reason is that a great macro cell UE power can resist the high intensity of interference from small cells, which can allow more small cell BSs deployment into the networks. However, the interference caused by macro cells also becomes high in intensity as the power continually increases, which leads to a decline in small cells' greatest ASR. In addition, regarding lower macro cell UE density, the peak amongst the small cells' greatest ASR may be obtained since less disturbance against small cells is generated from the low UE of macro cells.

Last, Fig. 8 illustrates the relationship between the maximum ASR, uplink power, and downlink power in small cells. From this figure, we can see the performance gain 


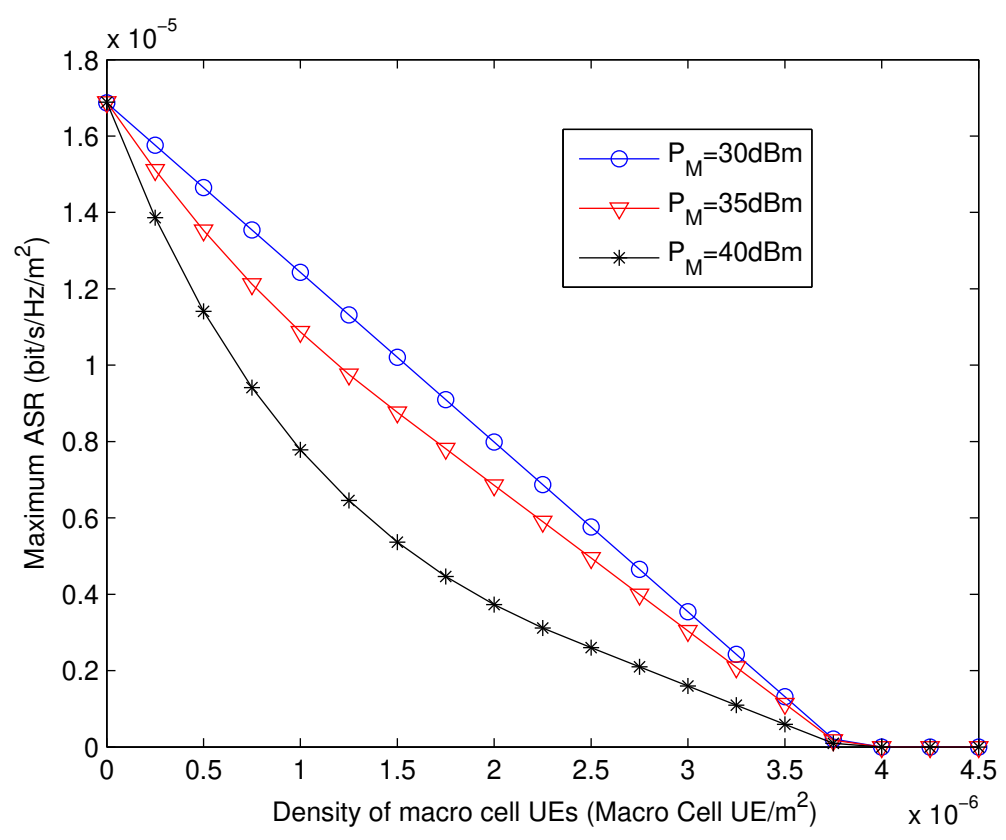

Figure 6 Maximum ASR of small cells vs. The density of macro cell UE

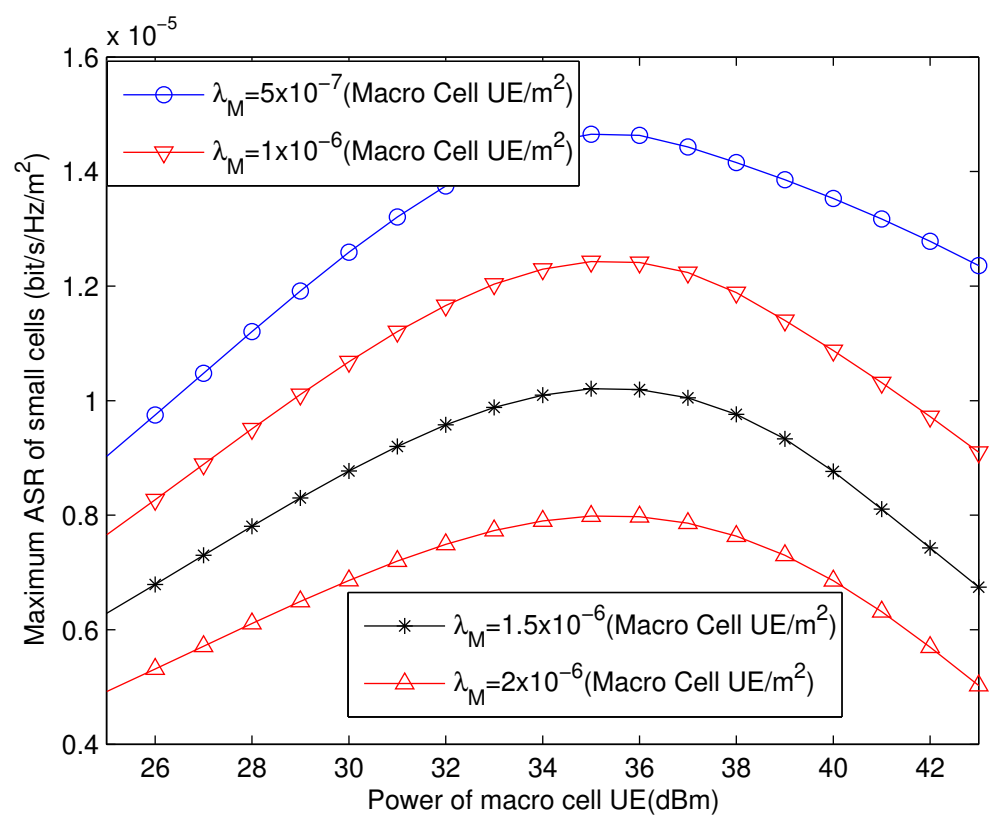

Figure 7 Maximum ASR of small cells vs. Power of macro cell UE 


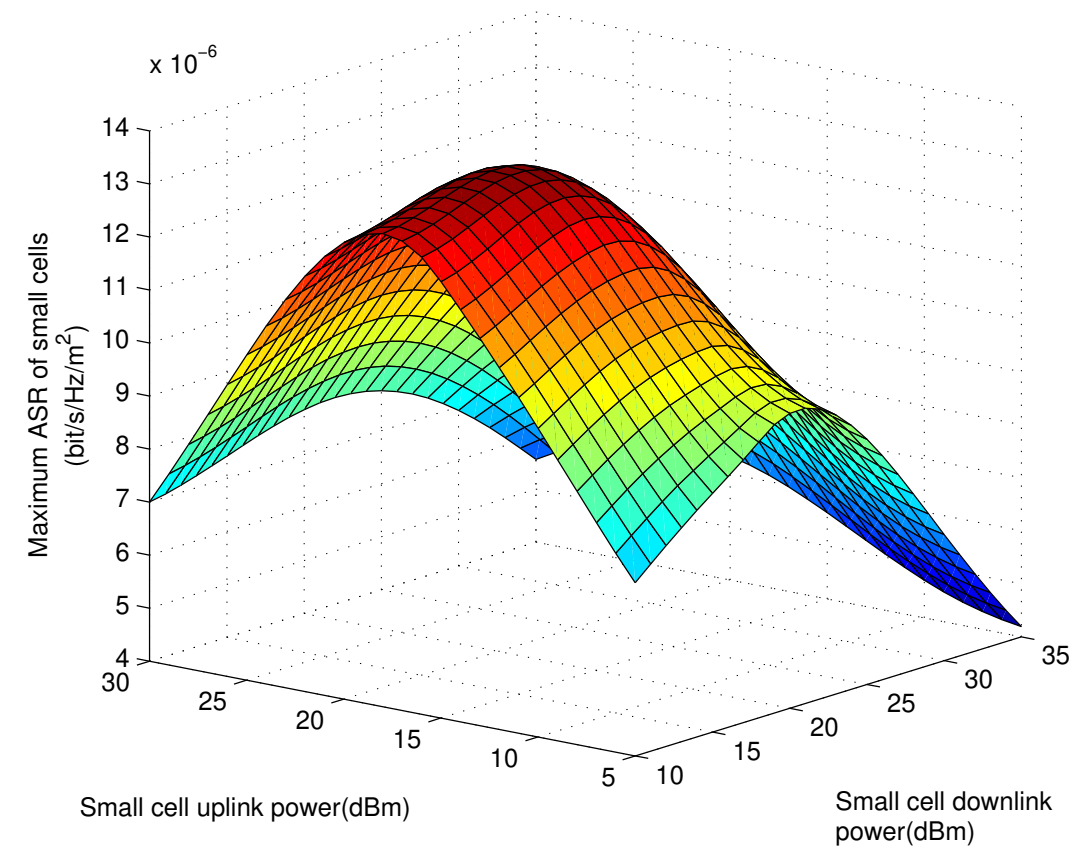

Figure 8 Maximum ASR of small cells vs. small cell uplink and downlink power

from uplink communication is larger than that of downlink communication. This is due to the uplink communication distance shorter than the distance of downlink communication, which the signal suffers from less propagation loss in the process of transmission. Furthermore, the probabilities of uplink and downlink transmission are set as 0.6 and 0.4 , respectively, which means that the number of links in the uplink transmission is more than that of downlink transmission. Hence, we can see that uplink communication contributes more to the enhancement of entire performance. An extreme condition in this case is that the lowest value for maximum ASR of small cells can be obtained when uplink and downlink transmit power are $5 \mathrm{dBm}$ and $35 \mathrm{dBm}$, respectively. This can be attributed to fact that uplink communication cannot enhance much performance of small cells. In addition, downlink communication will also cause much more interference to the entire networks.

\section{Conclusion}

This paper obtains BS density optimization amongst small cells, thus providing for their ASR maximization. Considering principles of stochastic geometry, the entire networks are model by homogeneous PPPs. Then, the probability of successful transmission in small and large cell systems is attained. Further, we evaluate small cells' expressions of ASR, and their BS density optimization has been generated, warranting both outage probability and density thresholds. Moreover, we have verified that the maximum ASR of small cells locates within fixed distances relative to their BS densities. Likewise, the boundaries of the density are also showed. Eventually, closed-form type of optimal density of small cells is obtained, from which ASR maximization can be realized. From the simulation, we further discuss the 
effects pertaining to certain factors such like the power of UE of macro cells, the optimal density of the BS of small cells, and the probability of transmission through downlink and uplink. The results indicate that ASR maximization can be mutually influenced by certain limitations involving UDN interferences and systems of macro cells.

Abbreviations

loT: Internet of Things; UDN: Ultra-Dense Networks; BS: Base Station; ASR: Average Sum Rate; PPP: Poisson Point Process; UE: User Equipment.

\section{Acknowledgements}

We express our gratification to the unnamed reviewers for their time and significant recommendations, thus helping in enhancing the entire value and organization of this study.

Funding

This research was funded by the basic BUPT scientific research project (500418763), and partly funded by Beijing Natural Science Foundation (L182024), National Natural Science Foundation of China (61801035).

Availability of data and materials

The datasets used or analysed during the current study are available from the corresponding author on reasonable request.

Competing interests

The authors declare that they have no competing interests.

\section{Authors' contributions}

Y.H.L. made independent contribution in the task planning, experiments simulation and outcomes analysis; Y.Y. aided in the composition of study and funds organization; L.Z. helped with paper reviewing and editing; D.Z.H and J.Y. helped with the validation of ideas and grammar check. All authors have perused and assented to the manuscript's printed version.

\section{Author details}

School of Information and Communication Engineering, Beijing University of Posts and Telecommunications, Beijing, China.

References

1. Zander, J.: Beyond the ultra-dense barrier: Paradigm shifts on the road beyond 1000x wireless capacity. IEEE Wireless Communications 24(3), 96-102 (2017). doi:10.1109/MWC.2017.1500377WC

2. Chen, S., Ma, R., Chen, H., Zhang, H., Meng, W., Liu, J.: Machine-to-machine communications in ultra-dense networks-a survey. IEEE Communications Surveys Tutorials 19(3), 1478-1503 (2017). doi:10.1109/COMST.2017.2678518

3. Kamel, M., Hamouda, W., Youssef, A.: Performance analysis of multiple association in ultra-dense networks. IEEE Transactions on Communications 65(9), 3818-3831 (2017). doi:10.1109/TCOMM.2017.2706261

4. Filo, M., Foh, C.H., Vahid, S., Tafazolli, R.: Performance analysis of ultra-dense networks with regularly deployed base stations. IEEE Transactions on Wireless Communications 19(5), 3530-3545 (2020). doi:10.1109/TWC.2020.2974729

5. Cao, J., Peng, T., Qi, Z., Duan, R., Yuan, Y., Wang, W.: Interference management in ultradense networks: A user-centric coalition formation game approach. IEEE Transactions on Vehicular Technology 67(6), 5188-5202 (2018)

6. Khaturia, M., Jha, P., Karandikar, A.: Connecting the unconnected: Toward frugal $5 \mathrm{~g}$ network architecture and standardization. IEEE Communications Standards Magazine 4(2), 64-71 (2020). doi:10.1109/MCOMSTD.001.1900006

7. Liao, X., Shi, J., Li, Z., Zhang, L., Xia, B.: A model-driven deep reinforcement learning heuristic algorithm for resource allocation in ultra-dense cellular networks. IEEE Transactions on Vehicular Technology 69(1), 983-997 (2020). doi:10.1109/TVT.2019.2954538

8. Rahman, G.M.S., Peng, M., Yan, S., Dang, T.: Learning based joint cache and power allocation in fog radio access networks. IEEE Transactions on Vehicular Technology 69(4), 4401-4411 (2020). doi:10.1109/TVT.2020.2975849

9. Flint, I., Kong, H., Privault, N., Wang, P., Niyato, D.: Analysis of heterogeneous wireless networks using poisson hard-core hole process. IEEE Transactions on Wireless Communications 16(11), 7152-7167 (2017) doi:10.1109/TWC.2017.2740387

10. Canbolat, A.I., Fukawa, K.: Joint interference suppression and multiuser detection schemes for multi-cell wireless relay communications: A three-cell case. IEEE Transactions on Communications 66(4), 1399-1410 (2018). doi:10.1109/TCOMM.2017.2780246

11. Wang, X., Zhang, H., Tian, Y., Zhu, C., Leung, V.C.M.: Optimal distributed interference mitigation for small cell networks with non-orthogonal multiple access: A locally cooperative game. IEEE Access 6, 63107-63119 (2018). doi:10.1109/ACCESS.2018.2877181

12. Pan, C., Mehrpouyan, H., Liu, Y., Elkashlan, M., Arumugam, N.: Joint pilot allocation and robust transmission design for ultra-dense user-centric tdd c-ran with imperfect csi. IEEE Transactions on Wireless Communications 17(3), 2038-2053 (2018) 
13. Tabassum, H., Dawy, Z., Hossain, E., Alouini, M.-S.: Interference statistics and capacity analysis for uplink transmission in two-tier small cell networks: A geometric probability approach. IEEE transactions on wireless communications 13(7), 3837-3852 (2014)

14. Chen, X., Wu, C., Chen, T., Zhang, H., Liu, Z., Zhang, Y., Bennis, M.: Age of information aware radio resource management in vehicular networks: A proactive deep reinforcement learning perspective. IEEE Transactions on Wireless Communications 19(4), 2268-2281 (2020). doi:10.1109/TWC.2019.2963667

15. Addali, K.M., Bani Melhem, S.Y., Khamayseh, Y., Zhang, Z., Kadoch, M.: Dynamic mobility load balancing for $5 \mathrm{~g}$ small-cell networks based on utility functions. IEEE Access 7, 126998-127011 (2019). doi:10.1109/ACCESS.2019.2939936

16. Quer, G., Pappalardo, I., Rao, B.D., Zorzi, M.: Proactive caching strategies in heterogeneous networks with device-to-device communications. IEEE Transactions on Wireless Communications 17(8), 5270-5281 (2018)

17. Wang, M., Xu, C., Chen, X., Hao, H., Zhong, L., Wu, D.O.: Design of multipath transmission control for information-centric internet of things: A distributed stochastic optimization framework. IEEE Internet of Things Journal 6(6), 9475-9488 (2019). doi:10.1109/JIOT.2019.2929263

18. Liu, X., Jia, M.: Intelligent spectrum resource allocation based on joint optimization in heterogeneous cognitive radio. IEEE Transactions on Emerging Topics in Computational Intelligence 4(1), 5-12 (2020). doi:10.1109/TETCI.2018.2865630

19. Zhang, H., Yang, N., Huangfu, W., Long, K., Leung, V.C.M.: Power control based on deep reinforcement learning for spectrum sharing. IEEE Transactions on Wireless Communications 19(6), 4209-4219 (2020). doi:10.1109/TWC.2020.2981320

20. Yang, Y., He, D., He, J., Thapa, A., Dahal, A.: Optimal deployment for maximizing average sum rate of small cells in ultra-dense networks. In: 2018 IEEE 29th Annual International Symposium on Personal, Indoor and Mobile Radio Communications (PIMRC), pp. 1-5 (2018). IEEE

21. Kibria, M.G., Nguyen, K., Villardi, G.P., Liao, W., Ishizu, K., Kojima, F.: A stochastic geometry analysis of multiconnectivity in heterogeneous wireless networks. IEEE Transactions on Vehicular Technology 67(10), 9734-9746 (2018). doi:10.1109/TVT.2018.2863280

22. Baccelli, F., Blaszczyszyn, B.: Stochastic Geometry and Wireless Networks: Theory. Now Publishers Inc, ??? (2009)

23. Haenggi, M.: Stochastic Geometry for Wireless Networks. Cambridge University Press, ??? (2012)

24. Hu, R.Q., Qian, Y.: Heterogeneous Cellular Networks. John Wiley \& Sons, ??? (2013)

25. Chisci, G., ElSawy, H., Conti, A., Alouini, M., Win, M.Z.: Latency in downlink cellular networks with random scheduling. In: ICC 2019 - 2019 IEEE International Conference on Communications (ICC), pp. 1-6 (2019). doi:10.1109/ICC.2019.8761076

26. Lee, C., Chang, R.Y., Lin, C., Cheng, S.: Energy-efficient d2d underlaid mimo cellular networks with energy harvesting. In: 2018 IEEE Global Communications Conference (GLOBECOM), pp. 1-7 (2018). doi:10.1109/GLOCOM.2018.8647272

27. Boyd, S., Vandenberghe, L.: Convex Optimization. Cambridge university press, ??? (2004) 
Figures

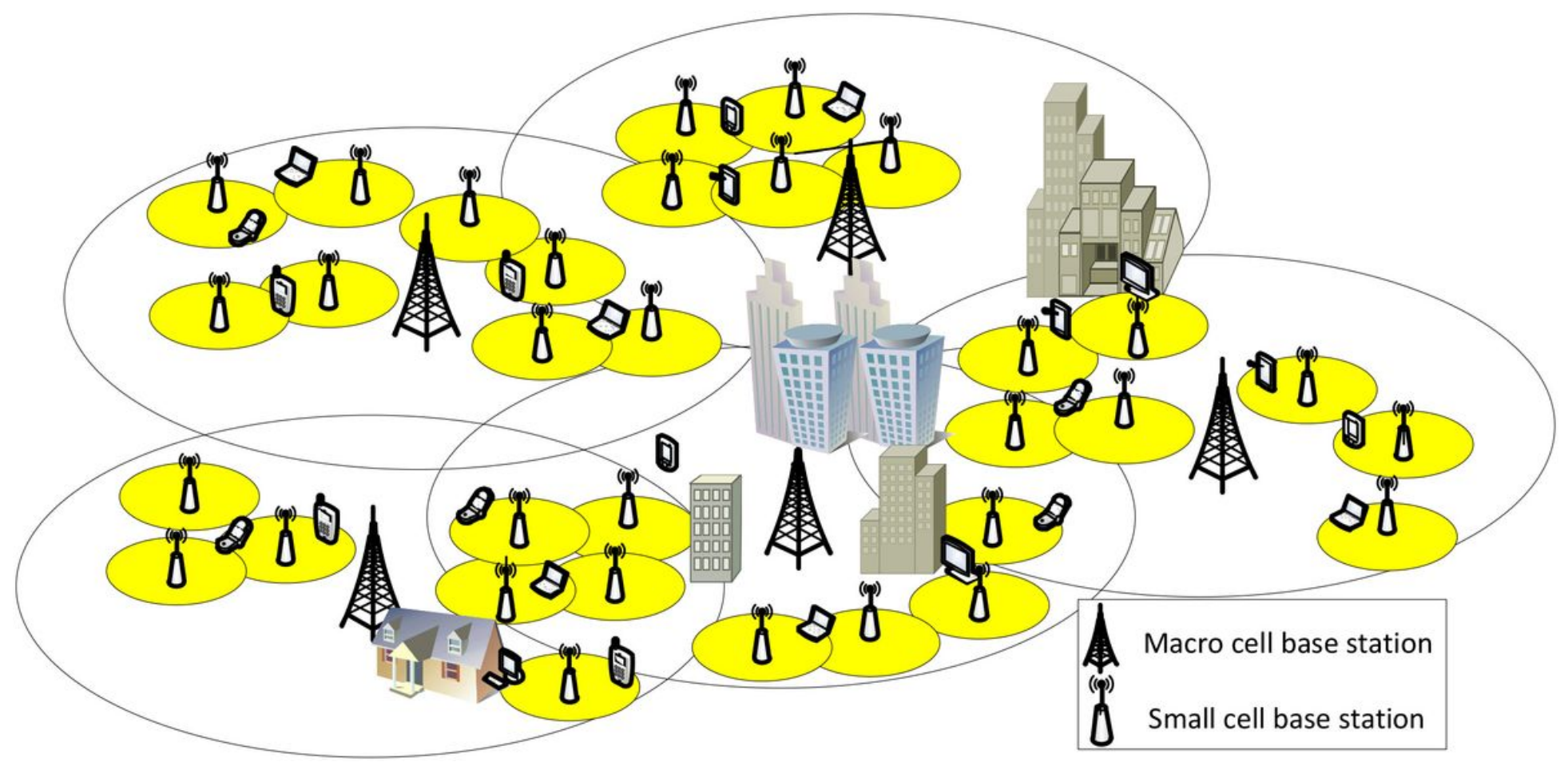

Figure 1

Scenario of UDN 


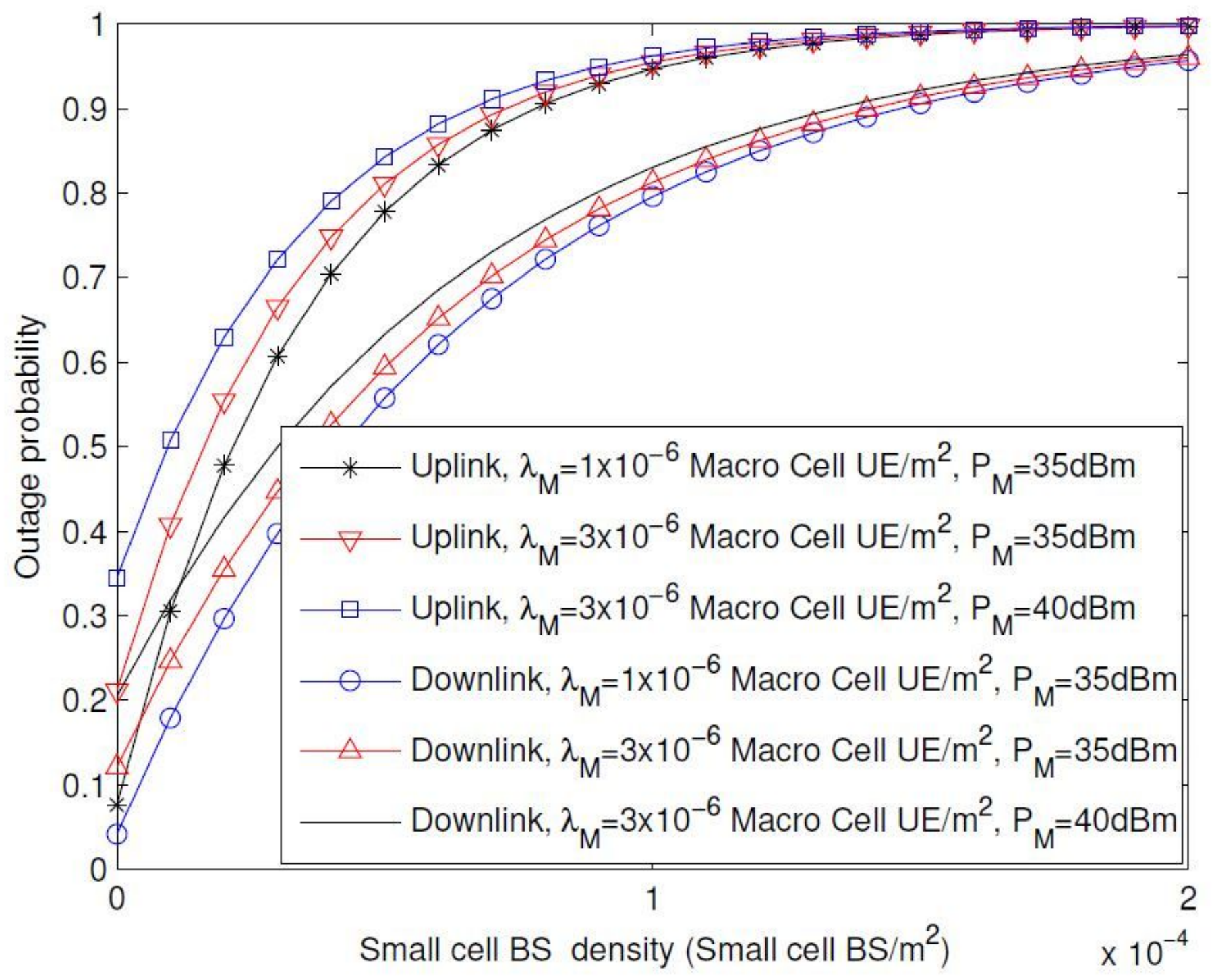

Figure 2

Outage probability in small cells 


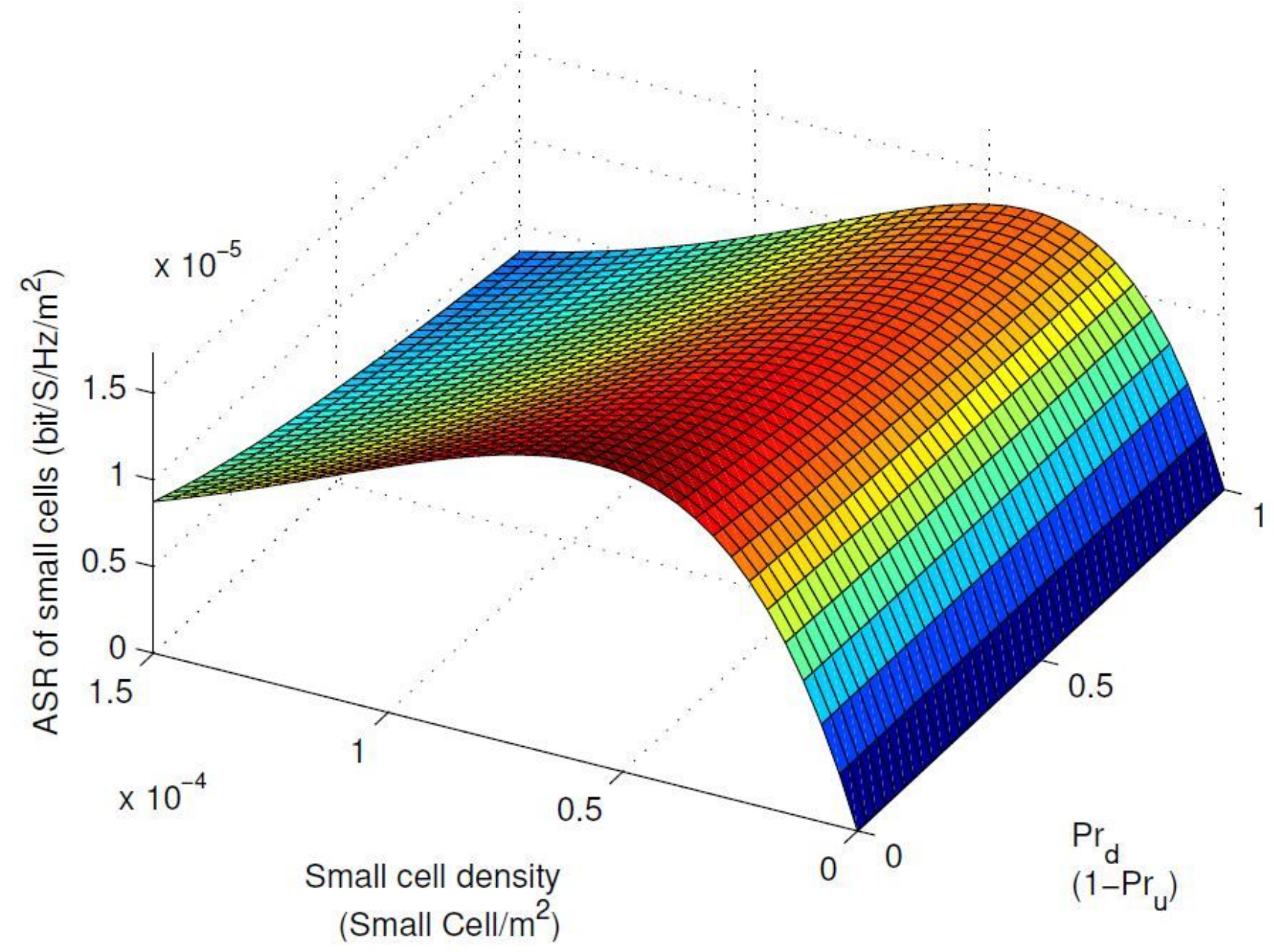

Figure 3

ASR of small cells vs. small cell density and probability of downlink 


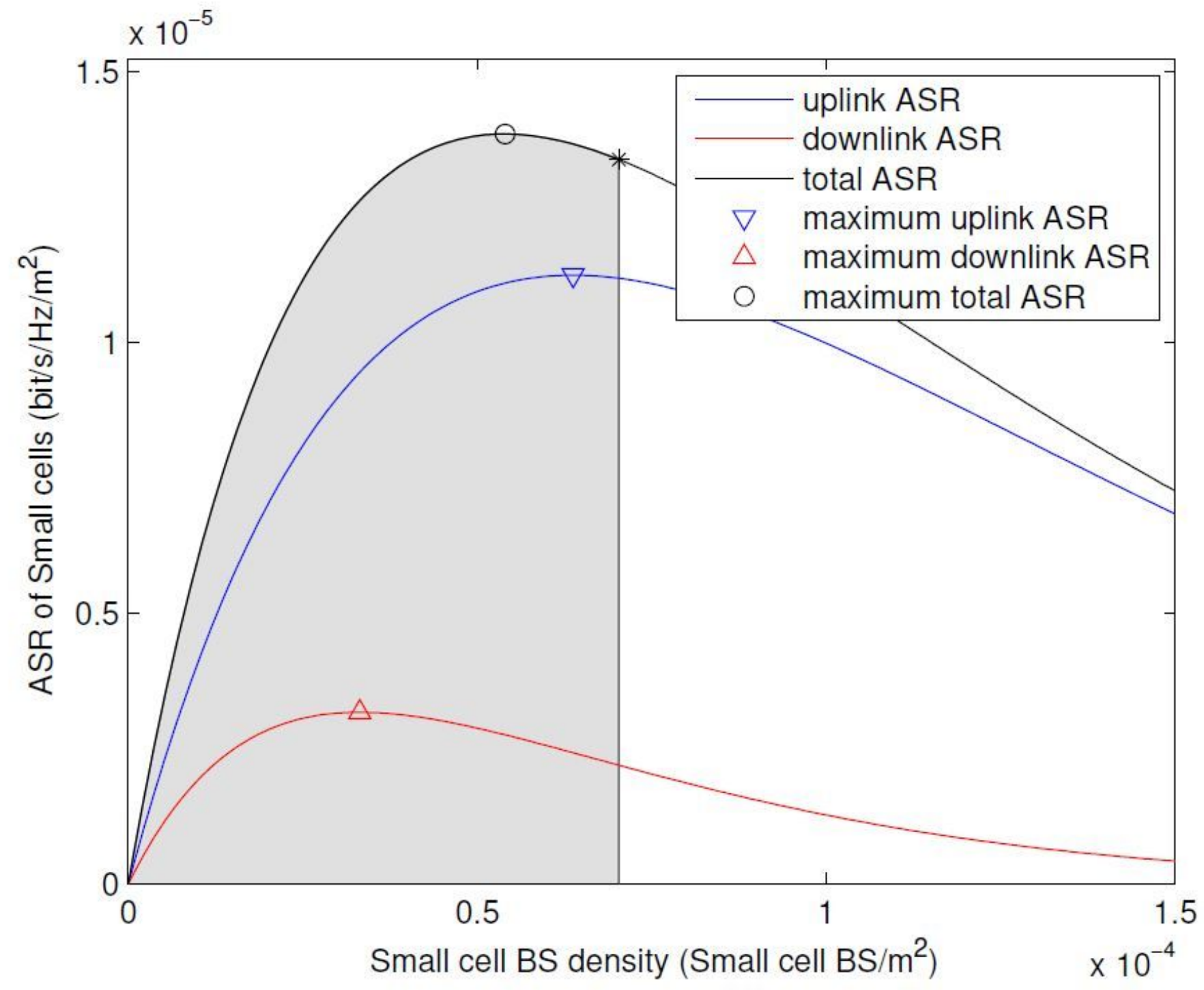

Figure 4

ASR of small cells vs. small cell BS density (Maximum ASR can be obtained) 


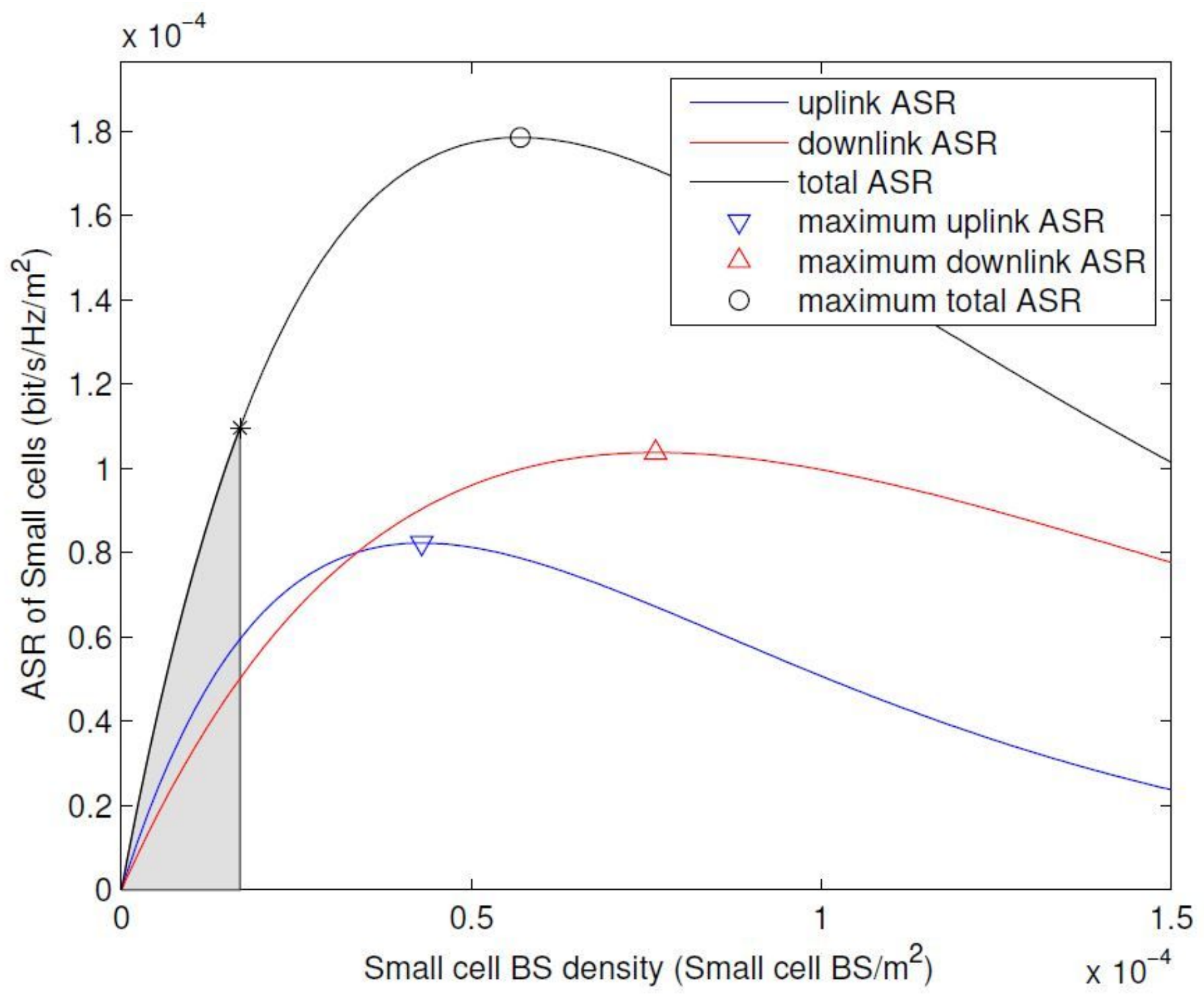

Figure 5

ASR of small cells vs. small cell BS density (Boundary value of ASR can be obtained) 


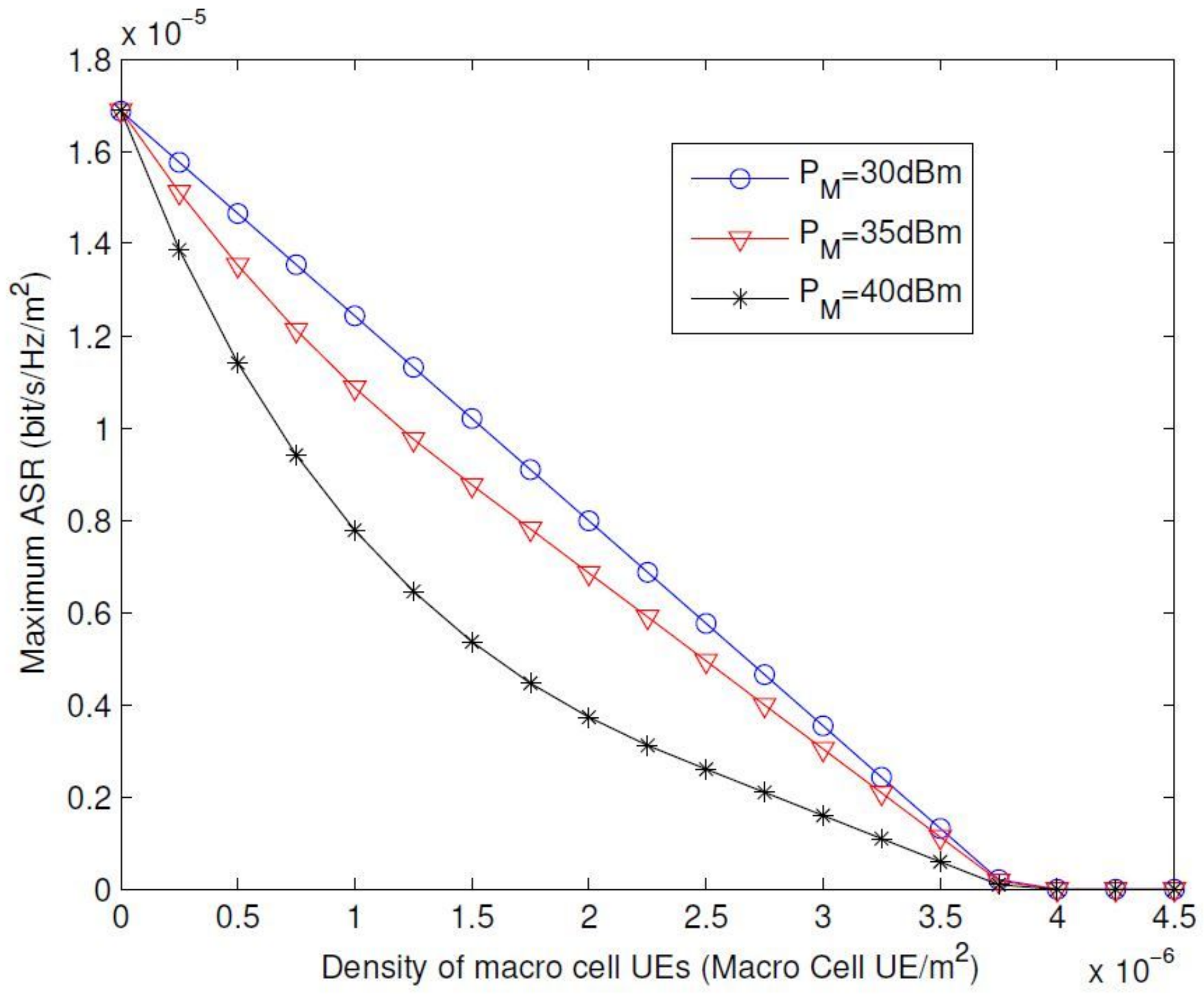

Figure 6

Maximum ASR of small cells vs. The density of macro cell UE 


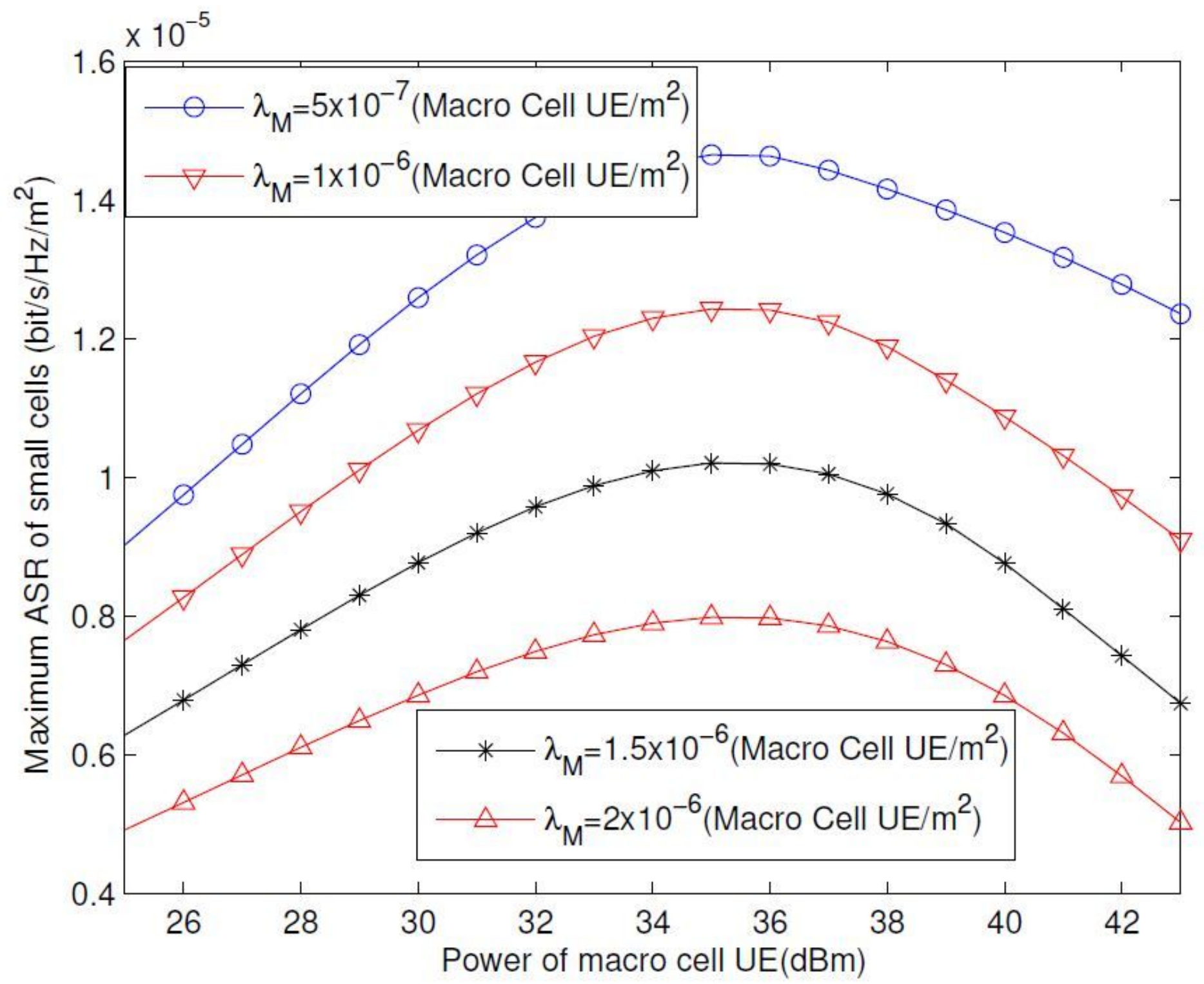

Figure 7

Maximum ASR of small cells vs. Power of macro cell UE 


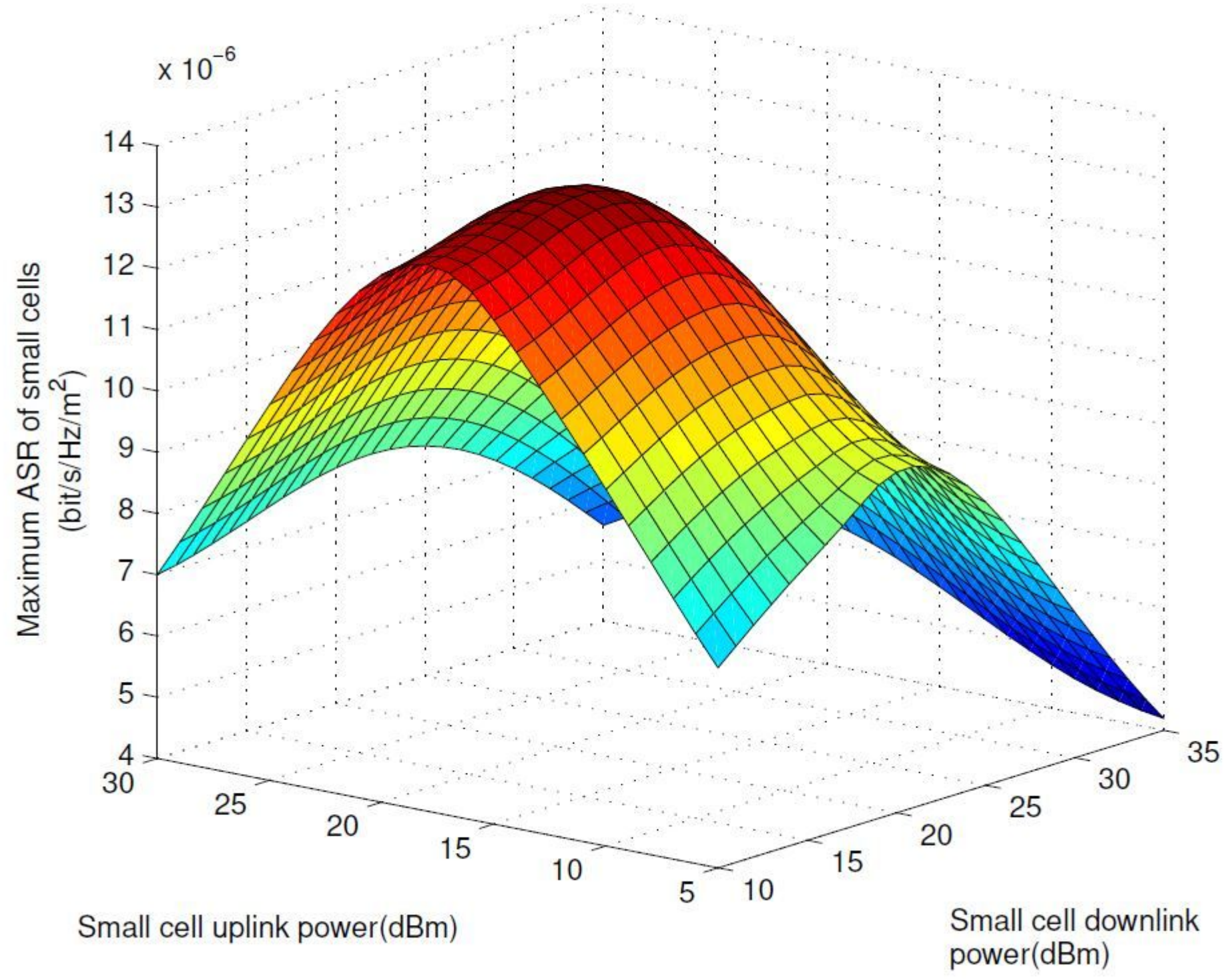

Figure 8

Maximum ASR of small cells vs. small cell uplink and downlink power 